\title{
Unsteady MHD Bionanofluid Flow in a Porous Medium with Thermal Radiation near a Stretching/Shrinking Sheet
}

\author{
M. Irfan, ${ }^{1}$ M. Asif Farooq $\mathbb{D}^{1},{ }^{1}$ A. Mushtaq $\mathbb{D}^{2},{ }^{2}$ and Z. H. Shamsi $\mathbb{C}^{3}$ \\ ${ }^{1}$ Department of Mathematics, School of Natural Sciences (SNS), National University of Sciences and Technology (NUST), \\ Sector H-12, Islamabad 44000, Pakistan \\ ${ }^{2}$ Seksjon for Matematikk, Nord Universitet, Bodø 8026, Norway \\ ${ }^{3}$ Department of Mathematics, University of the Punjab, Lahore 54590, Pakistan
}

Correspondence should be addressed to A. Mushtaq; asif.mushtaq@nord.no

Received 8 September 2020; Revised 14 October 2020; Accepted 21 October 2020; Published 16 November 2020

Academic Editor: Muhammad Mubashir Bhatti

Copyright ( ) 2020 M. Irfan et al. This is an open access article distributed under the Creative Commons Attribution License, which permits unrestricted use, distribution, and reproduction in any medium, provided the original work is properly cited.

\begin{abstract}
This research aims at providing the theoretical effects of the unsteady MHD stagnation point flow of heat and mass transfer across a stretching and shrinking surface in a porous medium including internal heat generation/absorption, thermal radiation, and chemical reaction. The fundamental principles of the similarity transformations are applied to the governing partial differential equations (PDEs) that lead to ordinary differential equations (ODEs). The transformed ODEs are numerically solved by the shooting algorithm implemented in MATLAB, and verification is done from MATLAB built-in solver $b v p 4 c$. The numerical data produced for the skin friction coefficient, the local Nusselt number, and the local Sherwood number are compared with the available result and found to be in a close agreement. The impact of involved physical parameters on velocity, temperature, concentration, and density of motile microorganisms profiles is scrutinized through graphs. It is analyzed that the skin friction coefficient enhances with increasing values of an unsteady parameter $A$, magnetic parameter $M$, and porosity parameter Kp. In addition, we observe that the density of a motile microorganisms profile enhances larger values of the bioconvection Lewis number $\mathrm{Lb}$ and Peclet number Pe and decreases with the increasing values of an unsteady parameter $A$.
\end{abstract}

\section{Introduction}

Nanofluids have been in demand because of its use in energy efficient devices due to its high performance contribution in thermal conductivity compared to a traditional fluid [1-3]. Nanofluids have recently been used in detergent, vehicle coolant, sensing in microelectromechanical systems (MEMS), and thermal energy storage [4]. Thus, it can be used in heating and electronic devices to make it more cost effective by minimization of energy lost in heat transfer process. There are a number of applications where nanofluids have been used such as in biomedical engineering, fluid power, mechanical and manufacturing industry, hydraulics, etc. The nanofluids are a composite solution containing nanoparticles and the base fluid [5].

The scope of nanofluid has been further enlarged by coalescing nanoparticles with blood to cultivate comprehension of biological sciences as well. Such a fluid is ordinarily known as bionanofluid. Recent applications of bionanofluid in medical sciences, such as medicine, cancer therapy, etc., have generated interest in investigating the bionanofluid flow. Moreover, the bionanofluid has instigated research in nanotechnology, biomedical engineering (applying biological in medical innovation), bioengineering (applying engineering principle to biology), and medical devices, etc.

Bioconvection is a process in which microorganisms convection occur in the fluid [6]. Khan and Makinde [7] investigated nanofluids in motile gyrotactic microorganisms. In [8], analytical solution of bioconvection of oxytactic bacteria was found. Mutuku and Makinde [9] discussed hydromagnetic bioconvection due to microorganisms and solution is obtained numerically. Recently, Naganthran et al. [10] applied extrapolation technique in time dependent 
bionanofluid. Zaimi et al. [11] discussed stagnation point flow not only containing nanoparticles but also gyrotactic microorganisms. Ali and Zaib [12] discussed unsteady flow of an Eyring-Powell nanofluid near a stagnation point. Zeng and Pedley [13] discussed gyrotactic microorganisms in complex three-dimensional flow. Shah et al. [14] have developed a fractional model in discussing a natural convection of bionanofluids between two vertical plates. Amirsom et al. [15] have discussed melting bioconvection nanofluid with second-order slip and thermal physical properties. Khader et al. [16] performed experimental study to determine the thermal and electrical conductivity to develop a new correlation in bionanofluid. For other details in this direction, see [17-22].

The thermal radiation plays an important role in industrial and engineering processes. Thermal radiation is a phenomenon in which energy is transported through indirect contact. Izadi et al. [23] discussed thermal radiation in a micropolar nanoliquid in a porous chamber. They applied the Galerkin finite element method to compute the numerical solution. Daniel et al. [24] presented a theory on entropy analysis for EMHD nanofluids considering thermal radiation and viscous dissipation. Muhammad et al. [25] obtained numerical solutions via the shooting method and bvp4c for the significant role nonlinear thermal radiation played in 3D Eyring-Powell nanofluid. Sohail et al. [26] described entropy analysis of Maxwell nanofluid in gyrotactic microorganisms with thermal radiation. Gireesha et al. [27] provide hybrid nanofluid flow across a permeable longitudinal moving fin with thermal radiation.

Eid [28] presents two-phase chemical reactions over a stretching sheet. Tripathy et al. [29] research chemical reactive flow over a moving vertical plate. In Pal and Talukdar [30], chemical reaction effects in a mixed convection flow have been covered. Katerina and Patel [31] reported results on radiation and chemical reaction in Casson fluid over an oscillating vertical plate. The works of Shah et al. [32], Rasool et al. [33], Khan et al. [34], and Khan et al. [35] contain chemical reactions as well as entropy generation over a nonlinear sheet. Khan et al. [36] present results on axisymmetric Carreau nanofluid along with chemical reaction. Gharami et al. [37] provide an unsteady flow of tangent nanofluid with a chemical reaction. Hamid et al. [38] simultaneously presented work on chemical reaction and activation energy in the unsteady flow of Williamson nanofluid. Reddy et al. [39] report results on nanofluid over a rotating disk with a chemical reaction. For other references on this topic, the reader is referred to [40-50].

In aforementioned literature studies, the chief emphasis has been made on various physical situations to find an indepth understanding of physics but the route of bionanofluid along with other situations of unsteady effect in a free stream flow is mostly absent from the literature.

The paper is written in the following order. Introduction of the paper is given in Section 1. Problem formulation is presented in Section 2. Numerical method is presented in Section 3. The results and discussion of the work are discussed Section 4. Conclusion is drawn at the end in Section 5.

\section{Problem Formulation}

Assuming an unsteady two-dimensional MHD stagnation point flow of bionanofluid in the presence of thermal radiation, chemical reaction, and internal heat generation/ absorption adjacent to a stretching sheet with thermal radiation, a water-based nanofluid containing nanoparticles and gyrotactic microorganisms is considered. It is assumed that the presence of nanoparticles has no effect on the swimming direction of microorganisms and on their swimming velocity. This assumption holds only for less than $1 \%$ concentration of nanoparticles. The magnetic Reynolds number of the flow is taken to be very small, so that the induced magnetic field is presumed to be negligible. The applied magnetic field $\beta_{o}^{2}$ is taken along the normal to the sheet. It is also assumed that the sheet is stretching/shrinking with a velocity $u_{e}=\epsilon \operatorname{ax}\left(1-A_{1} t\right)^{-1}, \epsilon>0$ indicates the stretching sheet whereas $\epsilon<0$ describes the shrinking sheet while $\epsilon=0$ represents a stationary sheet. The configuration of the flow is given in Figure 1.

Under the above assumptions, the governing model of flow reads as follows $[10,51]$ :

$$
\begin{aligned}
& \frac{\partial u}{\partial x}+\frac{\partial v}{\partial y}=0, \\
& \frac{\partial u}{\partial t}+u \frac{\partial u}{\partial x}+v \frac{\partial u}{\partial y}=u_{e} \frac{\partial u_{e}}{\partial x}+\frac{\partial u_{e}}{\partial t}+\nu \frac{\partial^{2} u}{\partial y^{2}}-\frac{\nu}{k^{*}}\left(u-u_{e}\right) \\
& \quad-\frac{\sigma \beta_{o}^{2}}{\rho}\left(u-u_{e}\right),
\end{aligned}
$$

$$
\begin{gathered}
\frac{\partial T}{\partial t}+u \frac{\partial T}{\partial x}+v \frac{\partial T}{\partial y}=\alpha \frac{\partial^{2} T}{\partial y^{2}}+\tau_{1}\left(D_{B} \frac{\partial T}{\partial y} \frac{\partial C}{\partial y}+\frac{D_{T}}{T_{\infty}}\left(\frac{\partial T}{\partial y}\right)^{2}\right) \\
-\frac{1}{\rho c_{p}} \frac{\partial q_{r}}{\partial y}+\frac{\mu}{\rho c_{p}}\left(\frac{\partial u}{\partial y}\right)^{2}+\frac{\left(T-T_{\infty}\right) Q}{\rho c_{p}}
\end{gathered}
$$

$$
\begin{aligned}
& \frac{\partial C}{\partial t}+u \frac{\partial C}{\partial x}+v \frac{\partial C}{\partial y}=D_{B} \frac{\partial^{2} C}{\partial y^{2}}+\frac{D_{T}}{T_{\infty}} \frac{\partial^{2} T}{\partial y^{2}}-\left(C-C_{\infty}\right) K_{c}, \\
& \frac{\partial N}{\partial t}+u \frac{\partial N}{\partial x}+v \frac{\partial N}{\partial y}+\frac{b w_{c}}{C_{w}-C_{\infty}}\left(\frac{\partial}{\partial y}\left(N \frac{\partial C}{\partial y}\right)\right)=D_{n} \frac{\partial^{2} N}{\partial y^{2}}
\end{aligned}
$$

However, the boundary conditions corresponding to the considered model is taken as follows: 


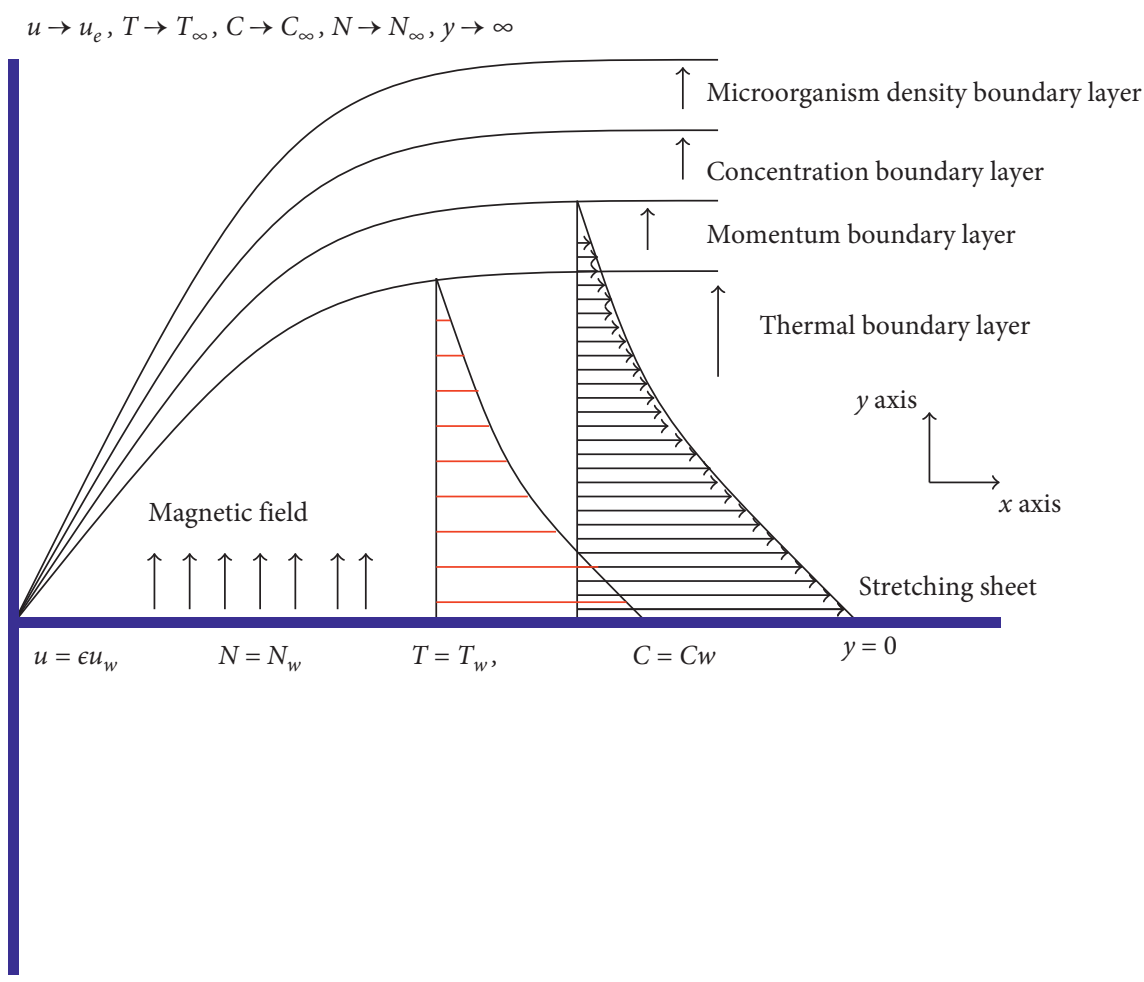

Figure 1: Structural diagram of the flow problem.

$$
\begin{aligned}
t \leq 0: & v=0, u=0, T=T_{\infty}, C=C_{\infty}, N=N_{\infty}, \\
t>0: & u=\epsilon u_{w}(x, t)=\epsilon \operatorname{ax}\left(1-A_{1} t\right)^{-1}, \quad \text { with } A_{1} t \neq 1, v=0, T=T_{w}, \\
C & =C_{w}, N=N_{w} \text { at } y=0, \\
u & =u_{e}(x, t)=\operatorname{ax}\left(1-A_{1} t\right)^{-1}, \quad \text { with } A_{1} t \neq 1, v=0, T=T_{\infty}, \\
C & =C_{\infty}, N=N_{\infty} \text { as } y \longrightarrow \infty
\end{aligned}
$$

where $t$ is time, $u, v$ are the velocity components in the $x-$ and $y$-axes, respectively. Furthermore, $T$ is a temperature of the fluid, $C$ is the concentration, $N$ is the density of the motile microorganisms, $k^{*}$ is the porosity of a porous medium, $\mu$ is the dynamic velocity of the fluid, $\sigma$ is the electrical conductivity of the fluid, $\rho$ is the density of the fluid, $\alpha$ is the thermal diffusivity, $c_{p}$ is the specific heat capacity at constant temperature, $\tau_{1}$ is the ratio of the effective heat capacity of the nanoparticle and the base fluid, $D_{B}$ is the Brownian diffusion coefficient, $D_{T}$ is thermophoretic diffusion coefficient, $D_{m}$ is the diffusivity of the microorganisms, $q_{r}$ is the radiative heat flux, $Q$ is the volumetric heat source, $K_{c}$ is called a rate of chemical reaction between the base fluid and nanoparticles, $W_{c}$ is the maximum cell swimming speed, and $b$ is the chemotaxis constant. Moreover, $T_{w}, C_{w}$, and $N_{w}$ are the temperature, nanoparticle concentration, and the density of the motile microorganisms at the wall and $T_{\infty}, C_{\infty}$, and $N_{\infty}$ are the temperature, nanoparticle concentration, and motile microorganisms far away from the sheet, respectively.
Introducing the similarity solutions as follows:

$$
\begin{aligned}
\eta & =\sqrt{\frac{a}{\nu\left(1-A_{1} t\right)} y,} \\
\psi & =\sqrt{\frac{\mathrm{a} v}{1-A_{1} t}} x f(\eta), \\
\theta(\eta) & =\frac{T-T_{\infty}}{T_{w}-T_{\infty}}, \\
\phi(\eta) & =\frac{C-C_{\infty}}{C_{w}-C_{\infty}}, \\
\chi(\eta) & =\frac{N-N_{\infty}}{N_{w}-N_{\infty}} .
\end{aligned}
$$

By inserting equation (7) into equations (1)-(5), we obtain the following transformed nonlinear ordinary differential equations: 


$$
\begin{array}{r}
f^{\prime \prime \prime}+f f^{\prime \prime}-f^{\prime 2}+1+A-A\left(f^{\prime}+\frac{\eta}{2} f^{\prime \prime}\right)-(M+\mathrm{Kp})\left(f^{\prime}-1\right)=0, \\
\left(1+\frac{4}{3} \mathrm{Rd}\right) \theta^{\prime \prime}+\operatorname{Pr} f \theta^{\prime}+\operatorname{Nb} \theta^{\prime} \phi^{\prime}+\mathrm{Nt} \theta^{\prime 2}+\operatorname{Pr}\left(\operatorname{Ec} f^{\prime \prime 2}+s \theta-\frac{\eta}{2} \theta^{\prime} A\right)=0, \\
\phi^{\prime \prime}+\frac{\mathrm{Nt}}{\mathrm{Nb}} \theta^{\prime \prime}+\operatorname{Le} \operatorname{Pr} f \phi^{\prime}-\frac{\eta}{2} \operatorname{Le} \operatorname{Pr} A \phi^{\prime}-\operatorname{Le} \operatorname{Pr} \operatorname{Kr} \phi=0, \\
\chi^{\prime \prime}+\operatorname{Lb} \operatorname{Pr} f \chi^{\prime}-\operatorname{Pe}\left(\phi^{\prime} \chi^{\prime}+\left(\chi+\sigma_{1}\right) \phi^{\prime \prime}\right)-\frac{\eta}{2} \operatorname{Lb} \operatorname{Pr} A \chi^{\prime}=0 .
\end{array}
$$

Similarly, equations (7) reduces boundary condition (6) into

$$
\begin{aligned}
f(0) & =0, f^{\prime}(0)=\epsilon, \theta(0)=1, \phi(0)=1, \chi(0)=1, \\
f^{\prime}(\infty) & =1, \theta(\infty)=0, \phi(\infty)=0, \chi(\infty)=0,
\end{aligned}
$$

where $A$ is an unsteadiness parameter, porous parameter $\mathrm{Kp}$, magnetic parameter $M$, Prandtl number Pr, thermal radiation parameter Rd, Brownian motion parameter Nb, thermophoretic parameter $\mathrm{Nt}$, Eckert number Ec, heat source parameter $s$, Lewis number Le, chemical reaction parameter $\mathrm{Kr}$, bioconvection Lewis number $\mathrm{Lb}$, Peclet number $\mathrm{Pe}$, and bioconvection parameter $\sigma_{1}$ are defined as follows:

$$
\begin{aligned}
& A=\frac{A_{1}}{a}, \\
& \mathrm{Kp}=\frac{\nu\left(1-A_{1} t\right)}{\mathrm{ak}^{*}}, \\
& M=\frac{\sigma B_{o}^{2}\left(1-A_{1} t\right)}{\rho a}, \\
& \operatorname{Pr}=\frac{\nu}{\alpha}, \\
& \mathrm{Rd}=\frac{4 \sigma T_{\infty}^{3}}{k_{1} k}, \\
& \mathrm{Nb}=\frac{\tau_{1} D_{B}\left(C_{w}-C_{\infty}\right)}{\alpha}, \\
& \mathrm{Nt}=\frac{\tau_{1} D_{T}\left(T_{w}-T_{\infty}\right)}{T_{\infty} \alpha}, \\
& \mathrm{Ec}=\frac{u_{e}^{2}}{c_{p}\left(T_{w}-T_{\infty}\right)}, \\
& s=\frac{Q\left(1-A_{1} t\right)}{a \rho c_{p}}, \\
& \text { Le }=\frac{\alpha}{D_{B}} \text {, } \\
& K_{r}=\frac{K_{c}\left(1-A_{1} t\right)}{a}, \\
& \mathrm{Lb}=\frac{\alpha}{D_{n}}, \\
& \mathrm{Pe}=\frac{\mathrm{bw}_{c}}{D_{n}}, \\
& \sigma_{1}=\frac{N_{\infty}}{N_{w}-N_{\infty}} \text {. }
\end{aligned}
$$

The physical quantities of interest in this study are the local skin friction coefficient $C_{\mathrm{fx}}$, the local Nusselt number $\mathrm{Nu}_{x}$, the local Sherwood number $\mathrm{Sh}_{x}$, and the local density number of motile microorganisms $\mathrm{Nn}_{x}$ are defined as follows:

$$
\begin{aligned}
& C_{f x}=\frac{\mu(\partial u / \partial y)_{y=0}}{\rho u_{e}^{2}}, \\
& \mathrm{Nu}_{x}= \frac{-k x(\partial T / \partial y)_{y=0}}{k\left(T_{w}-T_{\infty}\right)}, \\
& \mathrm{Sh}_{x}=\frac{-D_{B} x(\partial C / \partial y)_{y=0}}{D_{B}\left(C_{w}-C_{\infty}\right)}, \\
& \mathrm{Nn}_{x}=\frac{-D_{n} x(\partial N / \partial y)_{y=0}}{D_{n}\left(N_{w}-N_{\infty}\right)} .
\end{aligned}
$$

Inserting equation (7) into equation (11) yields the following expressions:

$$
\begin{aligned}
\operatorname{Re}_{x}^{(1 / 2)} C_{f x} & =f^{\prime \prime}(0), \\
\operatorname{Re}_{x}^{-(1 / 2)} \mathrm{Nu}_{x} & =-\left(1+\frac{4}{3} \mathrm{Rd}\right) \theta^{\prime}(0), \\
\operatorname{Re}_{x}^{-(1 / 2)} \mathrm{Sh}_{x} & =-\phi^{\prime}(0), \\
\operatorname{Re}_{x}^{-(1 / 2)} \mathrm{Nn}_{x} & =-\chi^{\prime}(0),
\end{aligned}
$$

where the local Reynolds number is defined as $\operatorname{Re}_{x}=\left(u_{e} x / \nu\right)$.

\section{Numerical Procedure}

3.1. Shooting Method. The physical model of ODEs alongside boundary conditions quantitatively evaluated by the shooting method implemented in MATLAB. The shooting approach involves two stages: Converting the boundary value problem (BVP) into an initial value problem (IVP) and the higher-order ODEs into a system of first-order ODEs. We employed the Newton-Raphson approach in locating roots. The Runge-Kutta method of order five is implemented in determining the solution of the IVP. The system of first-order ODEs reads as follows: 


$$
\begin{aligned}
& f=y_{1}, f^{\prime}=y_{2}, f^{\prime \prime}=y_{3}, f^{\prime \prime \prime}=y_{3}^{\prime}=-y_{1} y_{3}+y_{2}^{2}-1-A+A\left(y_{2}+\frac{\eta}{2} y_{3}\right)+(M+\mathrm{Kp})\left(y_{2}-1\right), \\
& y_{4}=\theta, y_{5}=\theta^{\prime}, \theta^{\prime \prime}=y_{5}^{\prime}=\frac{-1}{(1+(4 / 3) \mathrm{Rd})}\left(\operatorname{Pr} y_{1} y_{5}+\operatorname{Nb} y_{5} y_{7}+\operatorname{Nt} y_{5}^{2}+\operatorname{Pr}\left(\operatorname{Ec} y_{3}^{2}+s y_{4}-\frac{\eta}{2} A y_{5}\right)\right), \\
& y_{6}=\phi, y_{7}=\phi^{\prime}, \phi^{\prime \prime}=y_{7}^{\prime}=-\frac{N_{t}}{N_{b}} y_{5}^{\prime}-\operatorname{Le} \operatorname{Pr} y_{1} y_{7}+\frac{\operatorname{Le} \operatorname{Pr} \eta A}{2} y_{7}+\operatorname{Le} \operatorname{Pr} \operatorname{Kr} y_{6}, \\
& y_{8}=\chi, y_{9}=\chi^{\prime}, \chi^{\prime \prime}=y_{9}^{\prime}=-\operatorname{Lb} \operatorname{Pr} y_{1} y_{9}+\operatorname{Pe}\left(y_{7} y_{9}+\left(y_{8}+\sigma_{1}\right) y_{7}^{\prime}\right)+\frac{\eta}{2} \operatorname{Lb} \operatorname{Pr} A y_{9} .
\end{aligned}
$$

The converted form of boundary conditions into an initial condition for the shooting method is rewritten as follows:

$$
\begin{aligned}
& y_{1}(0)=0, \\
& y_{2}(0)=\epsilon, \\
& y_{4}(0)=1, \\
& y_{6}(0)=1, \\
& y_{8}(0)=1, \\
& y_{3}(0)=\tilde{\lambda}_{1}, \\
& y_{5}(0)=\tilde{\lambda}_{2}, \\
& y_{7}(0)=\tilde{\lambda}_{3}, \\
& y_{9}(0)=\tilde{\lambda}_{4} .
\end{aligned}
$$

3.2. bvp4c. Having found numerical results from the shooting method, we verify these results using MATLAB built-in solver bvp4c $[52,53]$. The bvp4c is a collocation solver which uses Gauss-Lobatto points to compute accurate results. In bvp4c, the first-order system of ODEs remains the same as discussed in Section 3.1. However, the boundary conditions implemented in MATLAB are as follows:

$$
\begin{gathered}
y_{1}(0)=0, \\
y_{2}(0)=\epsilon, \\
y_{4}(0)=1, \\
y_{6}(0)=1, \\
y_{8}(0)=1, \\
y_{2}(\infty)=1, \\
y_{4}(\infty)=0, \\
y_{6}(\infty)=0, \\
y_{8}(\infty)=0 .
\end{gathered}
$$

\section{Results and Discussion}

A summary of the current and the reported findings is seen with a minimal disparity in Table 1.

The data in Tables 2 and 3 show computational results for the skin friction coefficient, the local Nusselt number, the local Sherwood number, and the local density number of motile microorganisms obtained with the shooting method and the bvp4c. In Table 2, it is revealed that the skin friction coefficient $\mathrm{Cf}_{x}$ increases with increasing values of unsteady parameter $A$, magnetic parameter $M$, and porosity parameter Kp. However, decreasing trend is seen in the local Nusselt number $\mathrm{Nu}_{x}$ against an unsteady parameter $A$, radiation parameter $\mathrm{Rd}$, Brownian motion parameter $\mathrm{Nb}$, thermophoretic parameter $\mathrm{Nt}$, Eckert number Ec, and heat source parameter $s$. The local Nusselt number enhances the increasing values of Prandtl number Pr. The local Sherwood number $\mathrm{Sh}_{x}$ increases for higher values of Prandtl number $\mathrm{Pr}$, radiation parameter $\mathrm{Rd}$, Brownian motion parameter $\mathrm{Nb}$, Eckert number Ec, heat source parameter $s$, Lewis number Le, and chemical reaction parameter $\mathrm{Kr}$. The local Sherwood number decreases for higher values of thermophoretic parameter Nt. For the local density number of motile microorganisms, $\mathrm{Nn}_{x}$ shows decreasing trend for higher values of unsteady parameter $A$ and thermophoretic parameter $\mathrm{Nt}$ is observed while it increases by enhancing the Prandtl number $\mathrm{Pr}$, radiation parameter $\mathrm{Rd}$, Brownian motion parameter $\mathrm{Nb}$, Eckert number Ec, heat source parameter $s$, Lewis number Le, and chemical reaction parameter Kr. The local Sherwood number decreases for higher values of the thermophoretic parameter Nt. For the local density number of motile microorganisms, $\mathrm{Nn}_{x}$ shows decreasing trend for higher values of unsteady parameter $A$ and thermophoretic parameter $\mathrm{Nt}$ is observed while it increases by enhancing the Prandtl number $\mathrm{Pr}$, radiation parameter Rd, and Brownian motion parameter Nb,Eckert number Ec, heat source parameter $s$, Lewis number Le, chemical reaction parameter $\mathrm{Kr}$, the bioconvection Lewis number Lb, and Peclet number Pe.

In Figures 2 and 3, we present velocity profile results against parameters $M$ and Kp with $\epsilon=-0.5,0.5$ corresponding to shrinking and stretching sheets. In both cases, the boundary layer thickness decreases.

Figures 4-6 illustrate the impact of the Brownian motion parameter $\mathrm{Nb}$ on the temperature, concentration, and the density of motile microorganisms profiles for the case of stretching sheet $(\epsilon=-0.5)$ and shrinking sheet $(\epsilon=-0.5)$, respectively. Figure 4 gives an incremental thermal boundary layer thickness results as $\mathrm{Nb}$ increases. The thermal boundary layer thickness for the Brownian motion parameter with the stretching sheet is lower than the shrinking sheet. From Figure 5, it is observed that by increasing the Brownian motion parameter $\mathrm{Nb}$, the 
TABle 1: Comparison of the values of $f^{\prime \prime}(0),-\theta^{\prime}(0)$ and $-\phi^{\prime}(0)$ when $\epsilon=1, \mathrm{Le}=2, M=\mathrm{Kp}=A=\mathrm{Rd}=\mathrm{Ec}=s=\mathrm{Kr}=\mathrm{Lb}=\mathrm{Pe}=0$, $\mathrm{Nt}=\mathrm{Nb}=0.5$, and $\mathrm{Pr}=1$.

\begin{tabular}{lcccc}
\hline & Ibrahim et al. [51] & Zaimi et al. [11] & Naganthran et al. [10] & Present result (SM) \\
\hline$f^{\prime \prime}(0)$ & 0 & 0 & 0 & 0 \\
$-\theta^{\prime}(0)$ & 0.4767 & 0.476737 & 0.476737 & 0.4767 \\
$-\phi^{\prime}(0)$ & 1.0452 & 1.045154 & 1.045154 & 1.0452 \\
\hline
\end{tabular}

TABLE 2: Numerical values of $f^{\prime \prime}(0),-\theta^{\prime}(0),-\phi^{\prime}(0)$, and $-\chi^{\prime}(0)$ for several values of the involved parameter $A, M, \mathrm{Kp}, \mathrm{Pr}, \mathrm{Rd}, \mathrm{Nb}, \mathrm{Nt}, \mathrm{Ec}, s$, Le, Kr, Lb, Pe with $\epsilon=0.5$ and $\sigma_{1}=0.1$ (shooting method (SM)).

\begin{tabular}{|c|c|c|c|c|c|c|c|c|c|c|c|c|c|c|c|c|}
\hline & & & & & & & & & & & & & SM & SM & SM & SM \\
\hline$A$ & $M$ & $\mathrm{Kp}$ & $\operatorname{Pr}$ & $\mathrm{Rd}$ & $\mathrm{Nb}$ & $\mathrm{Nt}$ & $\mathrm{Ec}$ & $s$ & Le & $\mathrm{Kr}$ & $\mathrm{Lb}$ & $\mathrm{Pe}$ & $f^{\prime \prime}(0)$ & $-(1+(4 / 3) \mathrm{Rd}) \theta^{\prime}(0)$ & $-\phi^{\prime}(0)$ & $-\chi^{\prime}(0)$ \\
\hline 0.1 & 0.5 & 0.2 & 0.72 & 0.2 & 0.1 & 0.2 & 0.2 & 0.1 & 1 & 0.1 & 0.5 & 0.5 & 0.8364 & 0.5104 & 0.4108 & 0.5893 \\
\hline 0.3 & & & & & & & & & & & & & 0.8576 & 0.4687 & 0.4109 & 0.5658 \\
\hline 0.5 & & & & & & & & & & & & & 0.8784 & 0.4238 & 0.4121 & 0.5414 \\
\hline \multirow[t]{3}{*}{0.1} & 0.1 & 1 & 0.72 & 0.2 & 0.1 & 0.2 & 0.2 & 0.1 & 1 & 0.1 & 0.5 & 0.5 & 0.7749 & 0.5091 & 0.4082 & 0.5859 \\
\hline & 0.3 & & & & & & & & & & & & 0.8062 & 0.5098 & 0.4095 & 0.5876 \\
\hline & 0.5 & & & & & & & & & & & & 0.8364 & 0.5104 & 0.4108 & 0.5893 \\
\hline \multirow[t]{3}{*}{0.1} & 0.5 & 0 & 0.72 & 0.2 & 0.1 & 0.2 & 0.2 & 0.1 & 1 & 0.1 & 0.5 & 0.5 & 0.8062 & 0.5098 & 0.4095 & 0.5876 \\
\hline & & 0.3 & & & & & & & & & & & 0.8512 & 0.5107 & 0.4115 & 0.5900 \\
\hline & & 0.5 & & & & & & & & & & & 0.8799 & 0.5111 & 0.4127 & 0.5915 \\
\hline \multirow[t]{3}{*}{0.1} & 0.5 & 0.2 & 1 & 0.2 & 0.1 & 0.2 & 0.2 & 0.1 & 1 & 0.1 & 0.5 & 0.5 & 0.8364 & 0.5861 & 0.4919 & 0.6925 \\
\hline & & & 5 & & & & & & & & & & 0.8364 & 1.1092 & 1.1971 & 1.5280 \\
\hline & & & 10 & & & & & & & & & & 0.8364 & 1.4271 & 1.7646 & 2.1553 \\
\hline \multirow[t]{3}{*}{0.1} & 0.5 & 0.2 & 0.72 & 0 & 0.1 & 0.2 & 0.2 & 0.1 & 1 & 0.1 & 0.5 & 0.5 & 0.8364 & 0.4343 & 0.3812 & 0.5781 \\
\hline & & & & 0.3 & & & & & & & & & 0.8364 & 0.5456 & 0.4232 & 0.5940 \\
\hline & & & & 0.7 & & & & & & & & & 0.8364 & 0.6722 & 0.4611 & 0.6087 \\
\hline \multirow[t]{3}{*}{0.1} & 0.5 & 0.2 & 0.72 & 0.2 & 0.2 & 0.2 & 0.2 & 0.1 & 1 & 0.1 & 0.5 & 0.5 & 0.8364 & 0.4848 & 0.5386 & 0.6405 \\
\hline & & & & & 0.5 & & & & & & & & 0.8364 & 0.4132 & 0.6138 & 0.6709 \\
\hline & & & & & 0.7 & & & & & & & & 0.8364 & 0.3697 & 0.6272 & 0.6762 \\
\hline \multirow{3}{*}{0.1} & 0.5 & 0.2 & 0.72 & 0.2 & 0.1 & 0.1 & 0.2 & 0.1 & 1 & 0.1 & 0.5 & 0.5 & 0.8364 & 0.5282 & 0.5116 & 0.6269 \\
\hline & & & & & & 0.2 & & & & & & & 0.8364 & 0.5104 & 0.4108 & 0.5893 \\
\hline & & & & & & 0.4 & & & & & & & 0.8364 & 0.4765 & 0.2685 & 0.5453 \\
\hline \multirow[t]{3}{*}{0.1} & 0.5 & 0.2 & 0.72 & 0.2 & 0.1 & 0.2 & 0.2 & 0.1 & 1 & 0.1 & 0.5 & 0.5 & 0.8364 & 0.5104 & 0.4108 & 0.5893 \\
\hline & & & & & & & 0.4 & & & & & & 0.8364 & 0.4822 & 0.4518 & 0.6109 \\
\hline & & & & & & & 0.7 & & & & & & 0.8364 & 0.4398 & 0.5132 & 0.6434 \\
\hline \multirow[t]{3}{*}{0.1} & 0.5 & 0.2 & 0.72 & 0.2 & 0.1 & 0.2 & 0.2 & 0 & 1 & 0.1 & 0.5 & 0.5 & 0.8364 & 0.5701 & 0.3345 & 0.5508 \\
\hline & & & & & & & & 0.1 & & & & & 0.8364 & 0.5104 & 0.4108 & 0.5893 \\
\hline & & & & & & & & 0.2 & & & & & 0.8364 & 0.4468 & 0.4916 & 0.6299 \\
\hline \multirow[t]{3}{*}{0.1} & 0.5 & 0.2 & 0.72 & 0.2 & & 0.2 & 0.2 & 0.1 & 0.7 & 0.1 & 0.5 & 0.5 & 0.8364 & 0.5150 & 0.2662 & 0.5278 \\
\hline & & & & & & & & & 1 & & & & 0.8364 & 0.5104 & 0.4108 & 0.5893 \\
\hline & & & & & & & & & 1.3 & & & & 0.8364 & 0.5073 & 0.5261 & 0.6402 \\
\hline \multirow[t]{3}{*}{0.1} & 0.5 & 0.2 & 0.72 & 0.2 & 0.1 & 0.2 & 0.2 & 0.1 & 1 & 0.1 & 0.5 & 0.5 & 0.8364 & 0.5104 & 0.4108 & 0.5893 \\
\hline & & & & & & & & & & 0.4 & & & 0.8364 & 0.5075 & 0.6112 & 0.6861 \\
\hline & & & & & & & & & & 0.8 & & & 0.8364 & 0.5047 & 0.8266 & 0.7918 \\
\hline \multirow[t]{6}{*}{0.1} & 0.5 & 0.2 & 0.72 & 0.2 & 0.1 & 0.2 & & 0.1 & 1 & 0.1 & 0.5 & 0.5 & 0.8364 & 0.5104 & 0.4108 & 0.5893 \\
\hline & & & & & & & & & & & 1 & & 0.8364 & 0.5104 & 0.4108 & 0.7446 \\
\hline & & & & & & & & & & & 2 & & 0.8364 & 0.5104 & 0.4108 & 0.9578 \\
\hline & & & & & & & & & & 0.1 & 0.5 & 0.5 & 0.8364 & 0.5104 & 0.4108 & 0.5893 \\
\hline & & & & & & & & & & & & 1 & 0.8364 & 0.5104 & 0.4108 & 0.7629 \\
\hline & & & & & & & & & & & & 3 & 0.8364 & 0.5104 & 0.4108 & 1.5188 \\
\hline
\end{tabular}


TABle 3: Numerical values of $f^{\prime \prime}(0),-\theta^{\prime}(0),-\phi^{\prime}(0)$, and $-\chi^{\prime}(0)$ for several values of involved parameter $A, M, \mathrm{Kp}, \mathrm{Pr}, \mathrm{Rd}, \mathrm{Nb}, \mathrm{Nt}, \mathrm{Ec}, s, \mathrm{Le}$, $\mathrm{Kr}, \mathrm{Lb}, \mathrm{Pe}$ with $\epsilon=0.5$ and $\sigma_{1}=0.1$ (bvp4c).

\begin{tabular}{|c|c|c|c|c|c|c|c|c|c|c|c|c|c|c|c|c|}
\hline & & & & & & & & & & & & & bvp4c & bvp $4 c$ & bvp4c & bvp $4 c$ \\
\hline$A$ & $M$ & $\mathrm{Kp}$ & $\operatorname{Pr}$ & $\mathrm{Rd}$ & $\mathrm{Nb}$ & $\mathrm{Nt}$ & $\mathrm{Ec}$ & $s$ & Le & $\mathrm{Kr}$ & $\mathrm{Lb}$ & $\mathrm{Pe}$ & $f^{\prime \prime}(0)$ & $-(1+(4 / 3) \mathrm{Rd}) \theta^{\prime}(0)$ & $-\phi^{\prime}(0)$ & $-\chi^{\prime}(0)$ \\
\hline 0.1 & 0.5 & 0.2 & 0.72 & 0.2 & 0.1 & 0.2 & 0.2 & 0.1 & 1 & 0.1 & 0.5 & 0.5 & 0.8364 & 0.5104 & 0.4108 & 0.5893 \\
\hline 0.3 & & & & & & & & & & & & & 0.8576 & 0.4687 & 0.4108 & 0.5658 \\
\hline 0.5 & & & & & & & & & & & & & 0.8784 & 0.4238 & 0.4121 & 0.5414 \\
\hline \multirow[t]{3}{*}{0.1} & 0.1 & 1 & 0.72 & 0.2 & 0.1 & 0.2 & 0.2 & 0.1 & 1 & 0.1 & 0.5 & 0.5 & 0.7749 & 0.5091 & 0.4082 & 0.5859 \\
\hline & 0.3 & & & & & & & & & & & & 0.8062 & 0.5098 & 0.4095 & 0.5876 \\
\hline & 0.5 & & & & & & & & & & & & 0.8364 & 0.5104 & 0.4108 & 0.5893 \\
\hline \multirow[t]{3}{*}{0.1} & 0.5 & 0 & 0.72 & 0.2 & 0.1 & 0.2 & 0.2 & 0.1 & 1 & 0.1 & 0.5 & 0.5 & 0.8062 & 0.5098 & 0.4095 & 0.5876 \\
\hline & & 0.3 & & & & & & & & & & & 0.8512 & 0.5107 & 0.4115 & 0.5900 \\
\hline & & 0.5 & & & & & & & & & & & 0.8798 & 0.5111 & 0.4127 & 0.5915 \\
\hline \multirow[t]{3}{*}{0.1} & 0.5 & 0.2 & 1 & 0.2 & 0.1 & 0.2 & 0.2 & 0.1 & 1 & 0.1 & 0.5 & 0.5 & 0.8364 & 0.5861 & 0.4919 & 0.6925 \\
\hline & & & 5 & & & & & & & & & & 0.8364 & 1.1092 & 1.1971 & 1.5280 \\
\hline & & & 10 & & & & & & & & & & 0.8364 & 1.4271 & 1.7646 & 2.1553 \\
\hline \multirow[t]{3}{*}{0.1} & 0.5 & 0.2 & 0.72 & 0 & 0.1 & 0.2 & 0.2 & 0.1 & 1 & 0.1 & 0.5 & 0.5 & 0.8364 & 0.4343 & 0.3812 & 0.5781 \\
\hline & & & & 0.3 & & & & & & & & & 0.8364 & 0.5456 & 0.4232 & 0.5940 \\
\hline & & & & 0.7 & & & & & & & & & 0.8364 & 0.6722 & 0.4611 & 0.6087 \\
\hline \multirow[t]{3}{*}{0.1} & 0.5 & 0.2 & 0.72 & 0.2 & 0.2 & 0.2 & 0.2 & 0.1 & 1 & 0.1 & 0.5 & 0.5 & 0.8364 & 0.4848 & 0.5387 & 0.6405 \\
\hline & & & & & 0.5 & & & & & & & & 0.8364 & 0.4132 & 0.6138 & 0.6709 \\
\hline & & & & & 0.7 & & & & & & & & 0.8364 & 0.3697 & 0.6272 & 0.6762 \\
\hline \multirow[t]{3}{*}{0.1} & 0.5 & 0.2 & 0.72 & 0.2 & 0.1 & 0.1 & 0.2 & 0.1 & 1 & 0.1 & 0.5 & 0.5 & 0.8364 & 0.5282 & 0.5116 & 0.6269 \\
\hline & & & & & & 0.2 & & & & & & & 0.8364 & 0.5104 & 0.4108 & 0.5893 \\
\hline & & & & & & 0.4 & & & & & & & 0.8364 & 0.4764 & 0.2685 & 0.5453 \\
\hline \multirow[t]{3}{*}{0.1} & 0.5 & 0.2 & 0.72 & 0.2 & 0.1 & 0.2 & 0.2 & 0.1 & 1 & 0.1 & 0.5 & 0.5 & 0.8364 & 0.5104 & 0.4108 & 0.5893 \\
\hline & & & & & & & 0.4 & & & & & & 0.8364 & 0.4822 & 0.4518 & 0.6109 \\
\hline & & & & & & & 0.7 & & & & & & 0.8364 & 0.4398 & 0.5132 & 0.6434 \\
\hline \multirow[t]{3}{*}{0.1} & 0.5 & 0.2 & 0.72 & 0.2 & 0.1 & 0.2 & 0.2 & 0 & 1 & 0.1 & 0.5 & 0.5 & 0.8364 & 0.5701 & 0.3345 & 0.5508 \\
\hline & & & & & & & & 0.1 & & & & & 0.8364 & 0.5104 & 0.4108 & 0.5893 \\
\hline & & & & & & & & 0.2 & & & & & 0.8364 & 0.4467 & 0.4916 & 0.6299 \\
\hline \multirow[t]{3}{*}{0.1} & 0.5 & 0.2 & 0.72 & 0.2 & & 0.2 & 0.2 & 0.1 & 0.7 & 0.1 & 0.5 & 0.5 & 0.8364 & 0.5150 & 0.2662 & 0.5278 \\
\hline & & & & & & & & & 1 & & & & 0.8364 & 0.5104 & 0.4108 & 0.5893 \\
\hline & & & & & & & & & 1.3 & & & & 0.8364 & 0.5073 & 0.5261 & 0.6402 \\
\hline \multirow[t]{3}{*}{0.1} & 0.5 & 0.2 & 0.72 & 0.2 & 0.1 & 0.2 & 0.2 & 0.1 & 1 & 0.1 & 0.5 & 0.5 & 0.8364 & 0.5104 & 0.4108 & 0.5893 \\
\hline & & & & & & & & & & 0.4 & & & 0.8364 & 0.5075 & 0.6112 & 0.6861 \\
\hline & & & & & & & & & & 0.8 & & & 0.8364 & 0.5047 & 0.8266 & 0.7918 \\
\hline \multirow[t]{6}{*}{0.1} & 0.5 & 0.2 & 0.72 & 0.2 & 0.1 & 0.2 & & 0.1 & 1 & 0.1 & 0.5 & 0.5 & 0.8364 & 0.5104 & 0.4108 & 0.5893 \\
\hline & & & & & & & & & & & 1 & & 0.8364 & 0.5104 & 0.4108 & 0.7446 \\
\hline & & & & & & & & & & & 2 & & 0.8364 & 0.5104 & 0.4108 & 0.9578 \\
\hline & & & & & & & & & & 0.1 & 0.5 & 0.5 & 0.8364 & 0.5104 & 0.4108 & 0.5893 \\
\hline & & & & & & & & & & & & 1 & 0.8364 & 0.5104 & 0.4108 & 0.7629 \\
\hline & & & & & & & & & & & & 3 & 0.8364 & 0.5104 & 0.4108 & 1.5189 \\
\hline
\end{tabular}

concentration boundary layer thickness reduces in both stretching and shrinking sheet cases. Figure 6 exhibits that for higher values of the Brownian motion parameter $\mathrm{Nb}$, the density of motile microorganisms decreases. This decrease in the density of motile microorganisms is higher in the shrinking sheet case as compared to the stretching sheet case.

The impact of the thermophoresis parameter $\mathrm{Nt}$ on temperature, concentration, and density of motile microorganisms can be seen in Figures 7-9. Figure 7 reveals that the thermal boundary layer thickness increases for larger values of the thermophoresis parameter Nt. Figures 8 and 9 indicate that the concentration and density of motile microorganisms increases by increasing thermophoresis parameter $\mathrm{Nt}$, respectively.
Figure 10 depicts the behavior of a radiation parameter Rd on the temperature profile. We observe that by increasing radiation parameter, thermal boundary layer thickness increases in both stretching and shrinking sheet cases.

Figure 11 characterizes the influence of Eckert number Ec on temperature distribution. We conclude that increment in Eckert number Ec enhances the temperature profile.

Figure 12 scrutinizes the impact of the heat source parameter $s$ on the temperature profile. It is seen that for higher values of the heat source parameter $s$, the temperature profile increases.

Figure 13 examines the effect of the Prandtl number Pr on the temperature profile. We analyzed that enhancement in Prandtl number $\operatorname{Pr}$ causes a reduction in thermal boundary layer thickness. 


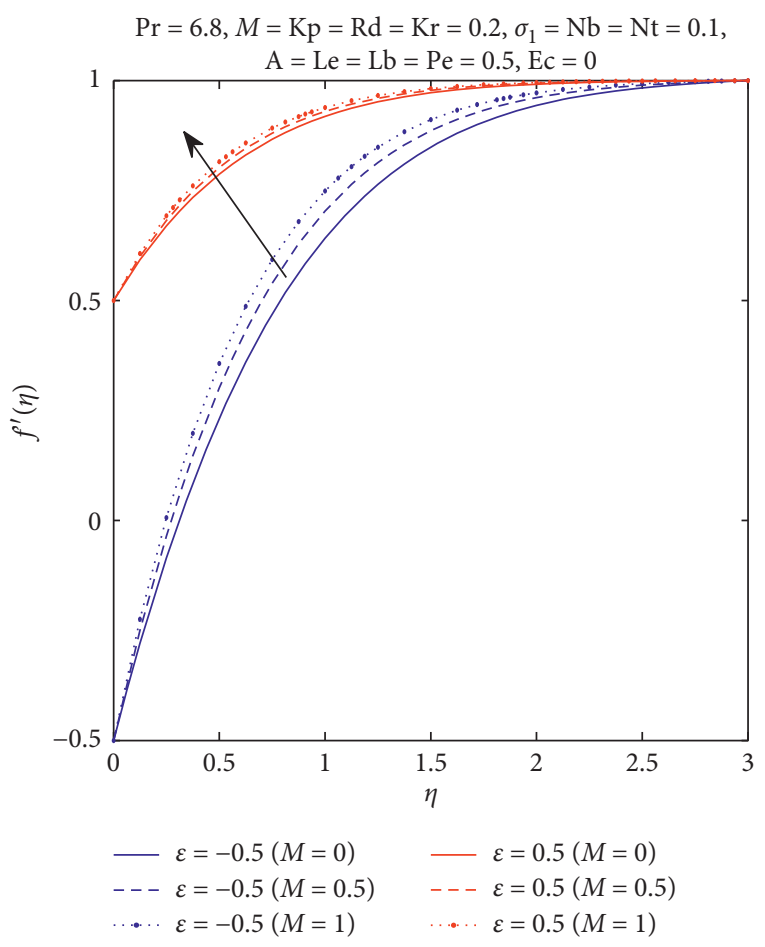

Figure 2: Velocity profile $f^{\prime}(\eta)$ for different $M$.

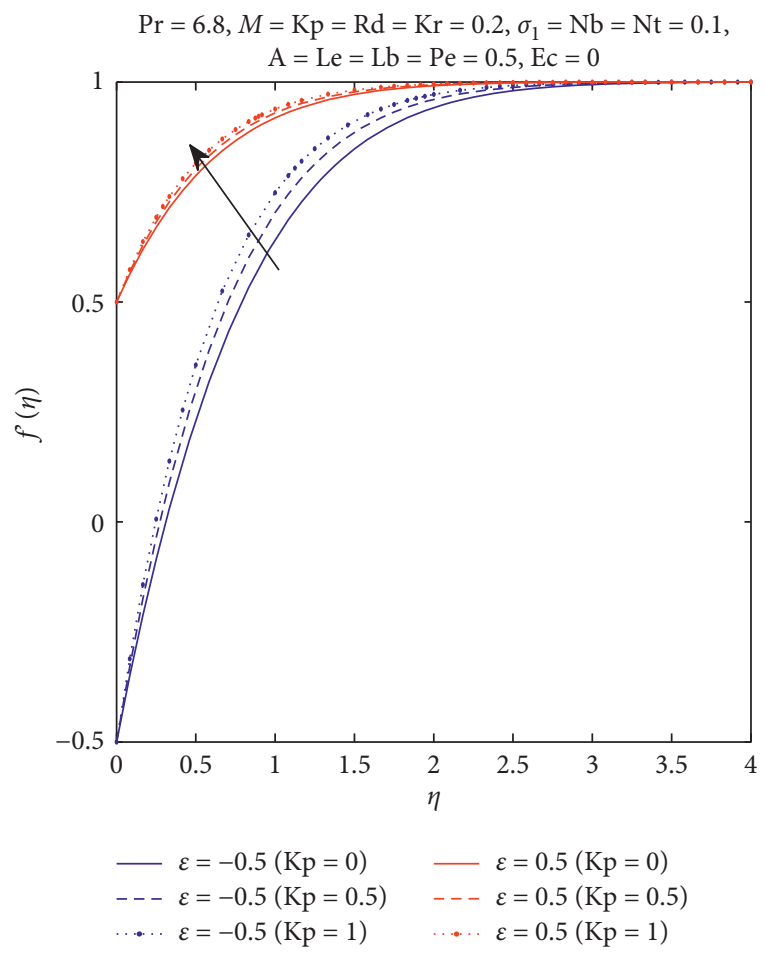

Figure 3: Velocity profile $f^{\prime}(\eta)$ for different Kp.

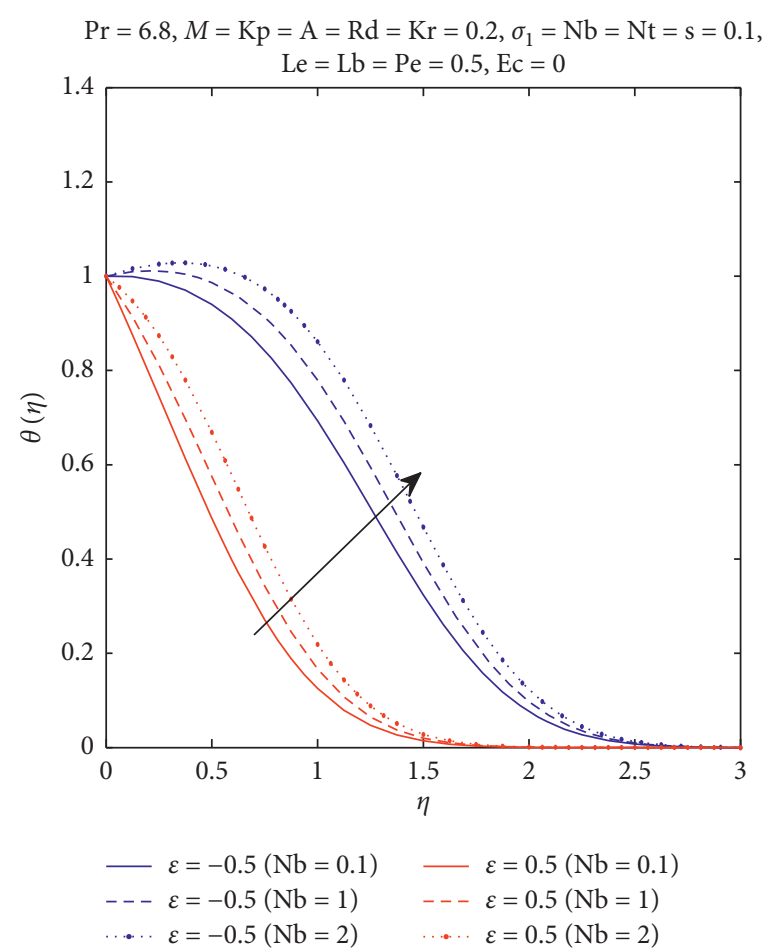

Figure 4: Temperature profile $\theta(\eta)$ for different $\mathrm{Nb}$.

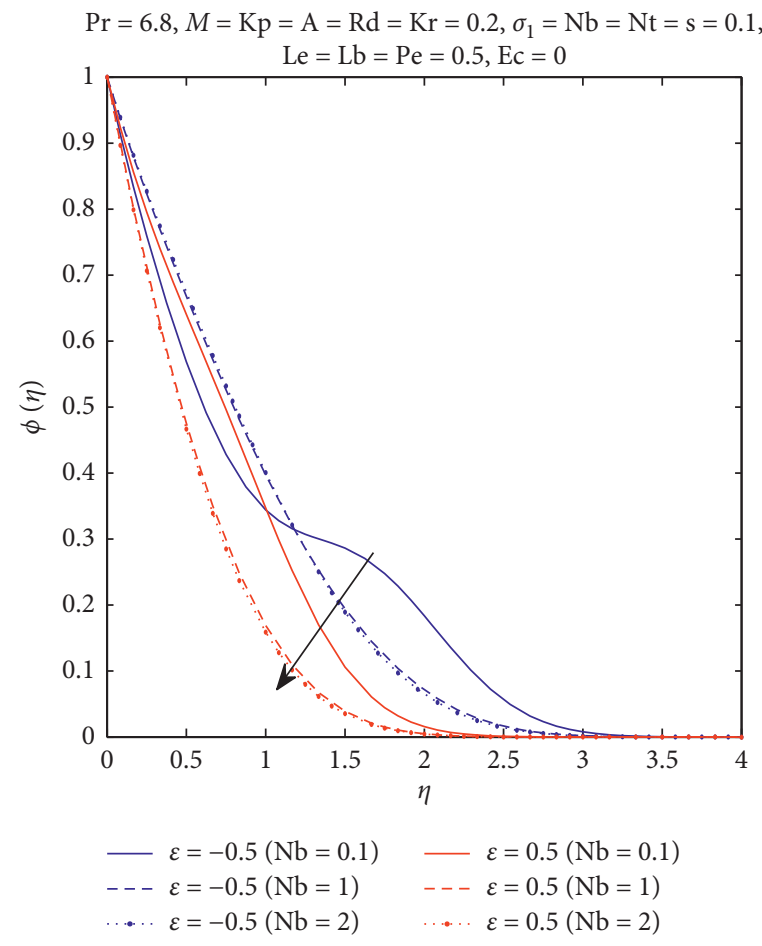

Figure 5: Concentration profile $\phi(\eta)$ for different $\mathrm{Nb}$. 


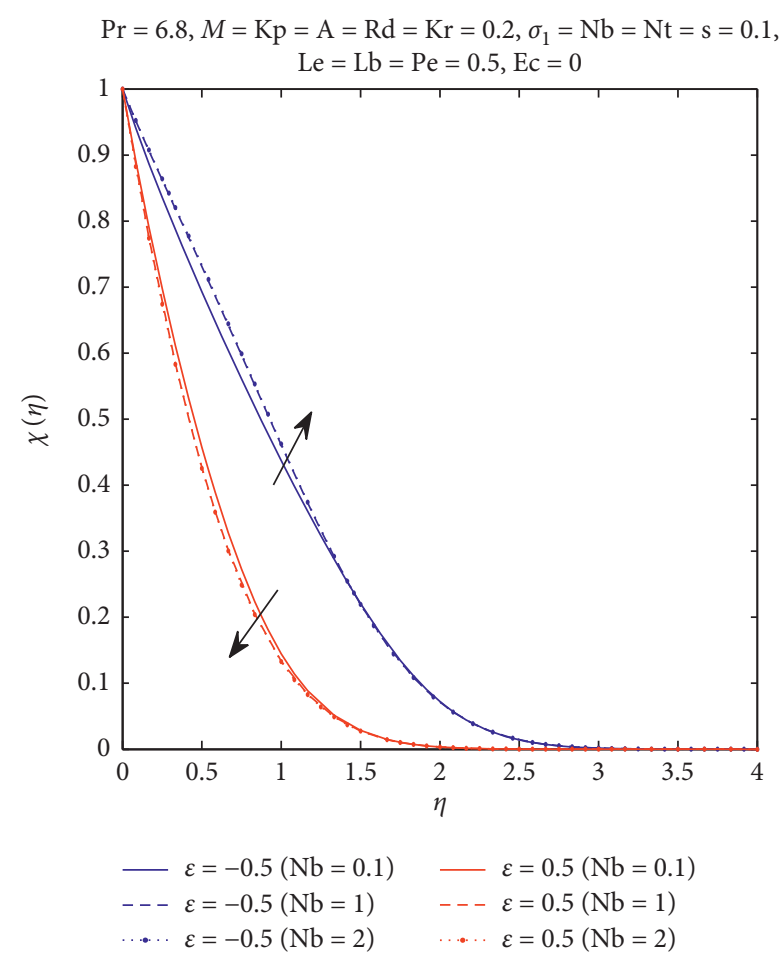

Figure 6: Microorganisms profile $\chi(\eta)$ for different $\mathrm{Nb}$.

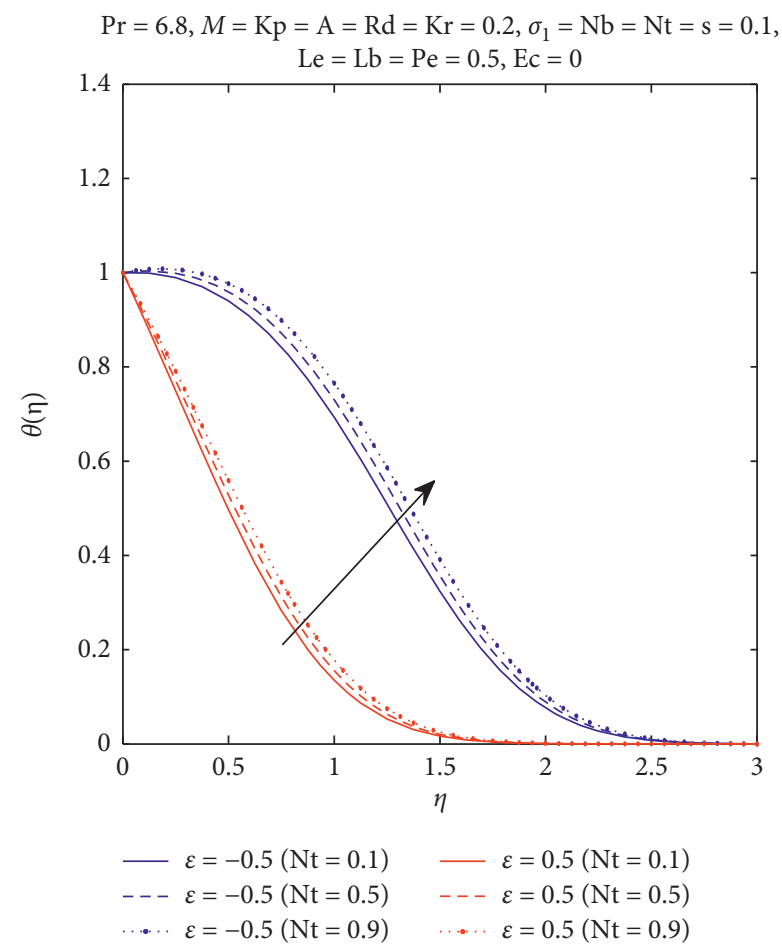

FIgURE 7: Temperature profile $\theta(\eta)$ for different Nt.

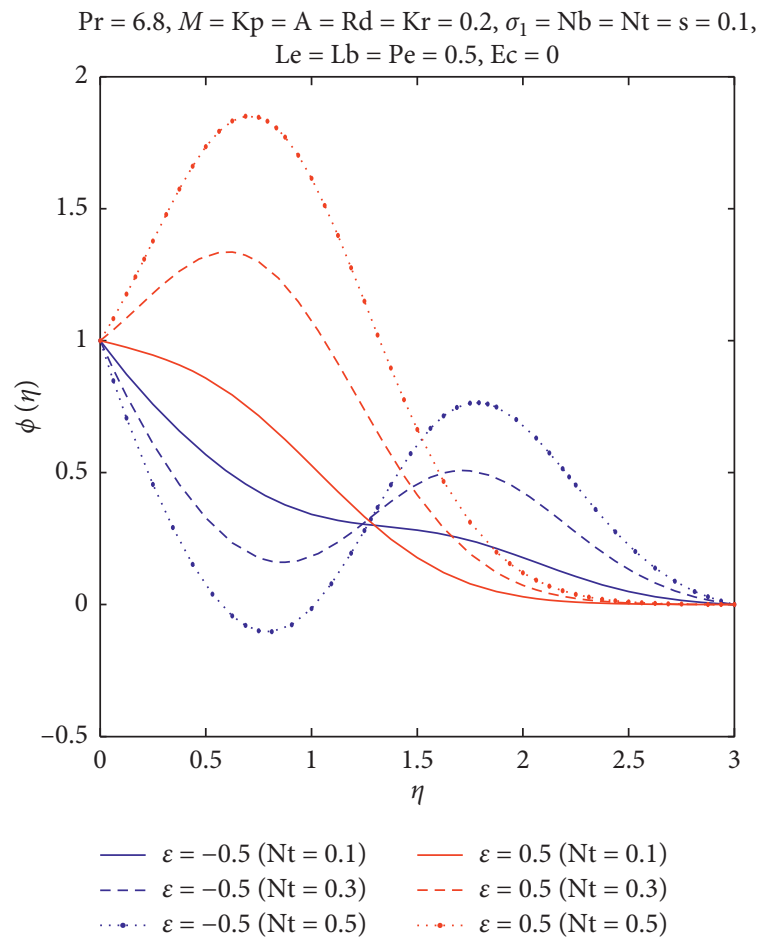

Figure 8: Concentration profile $\phi(\eta)$ for different Nt.

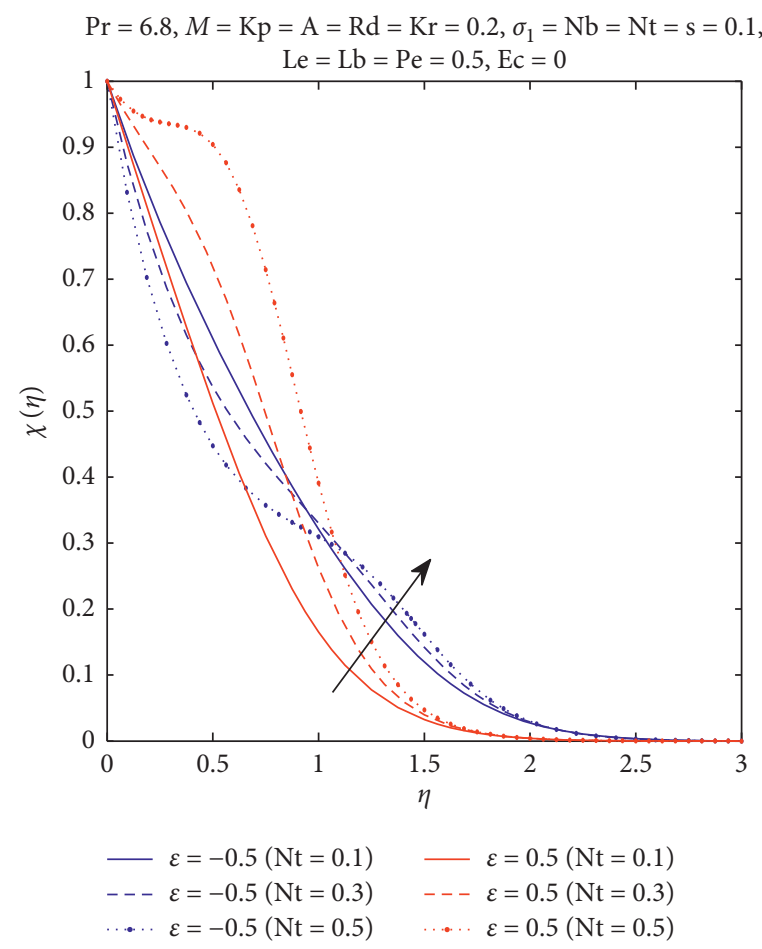

Figure 9: Microorganisms profile $\chi(\eta)$ for different Nt. 


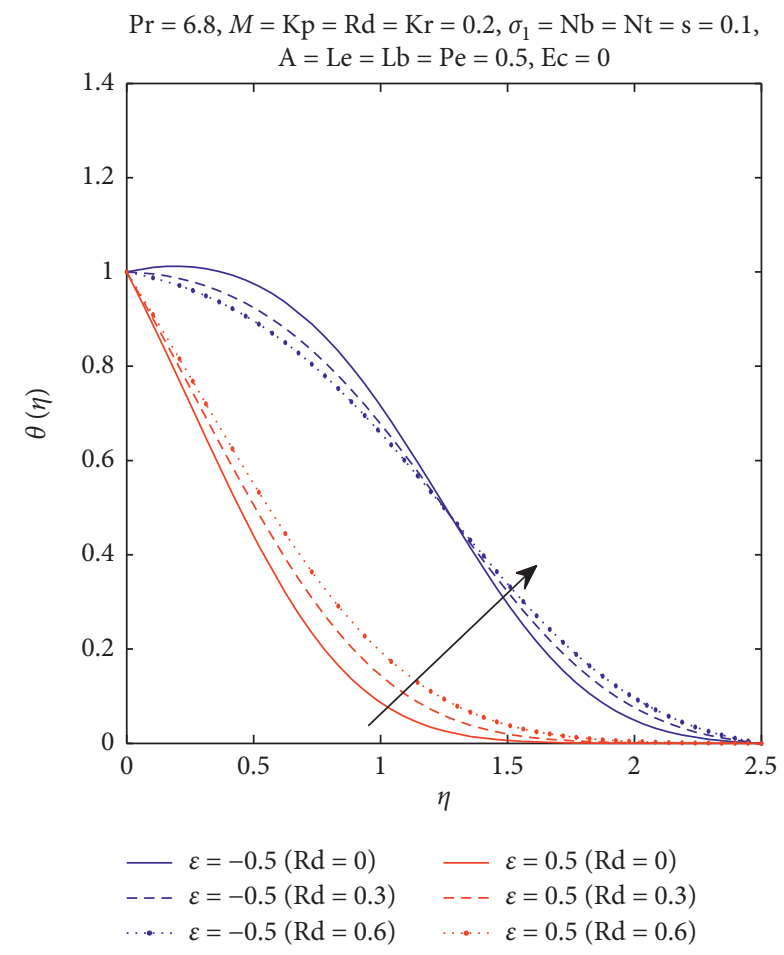

FIgURE 10: Temperature profile $\theta(\eta)$ for different $\mathrm{Rd}$.

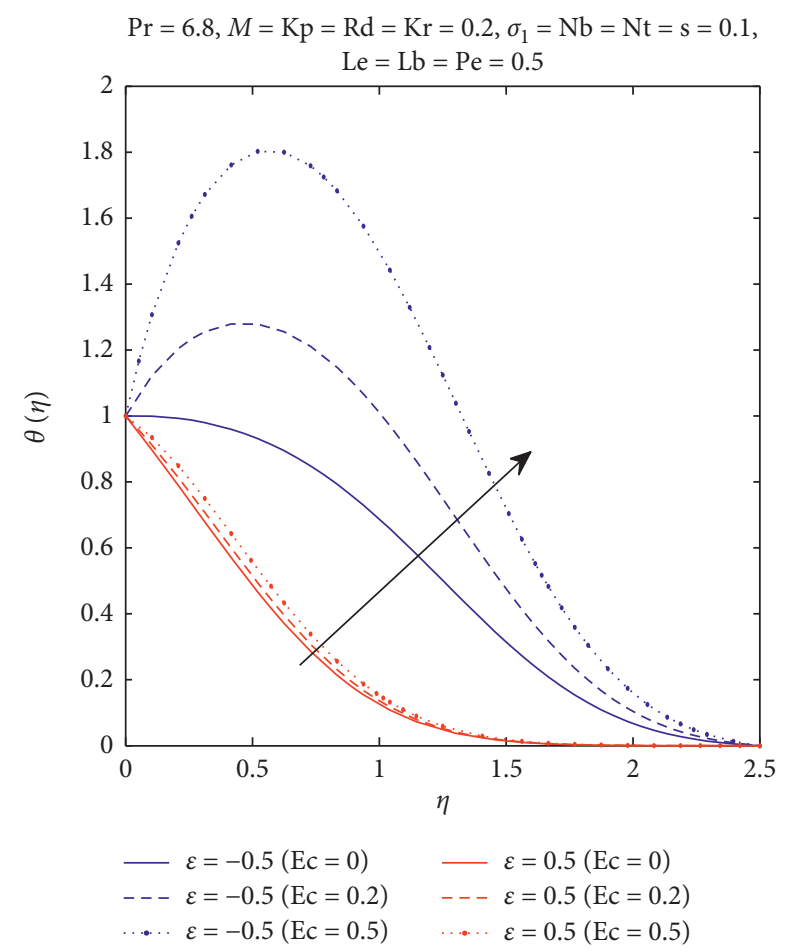

FIgURE 11: Temperature profile $\theta(\eta)$ for different Ec.

Figure 14 is drawn to perceive the impact of bioconvection Lewis number Lb on the density of motile microorganisms profile. It is observed that higher values of bioconvection Lewis number Lb lower the boundary layer thickness of motile microorganisms profile.

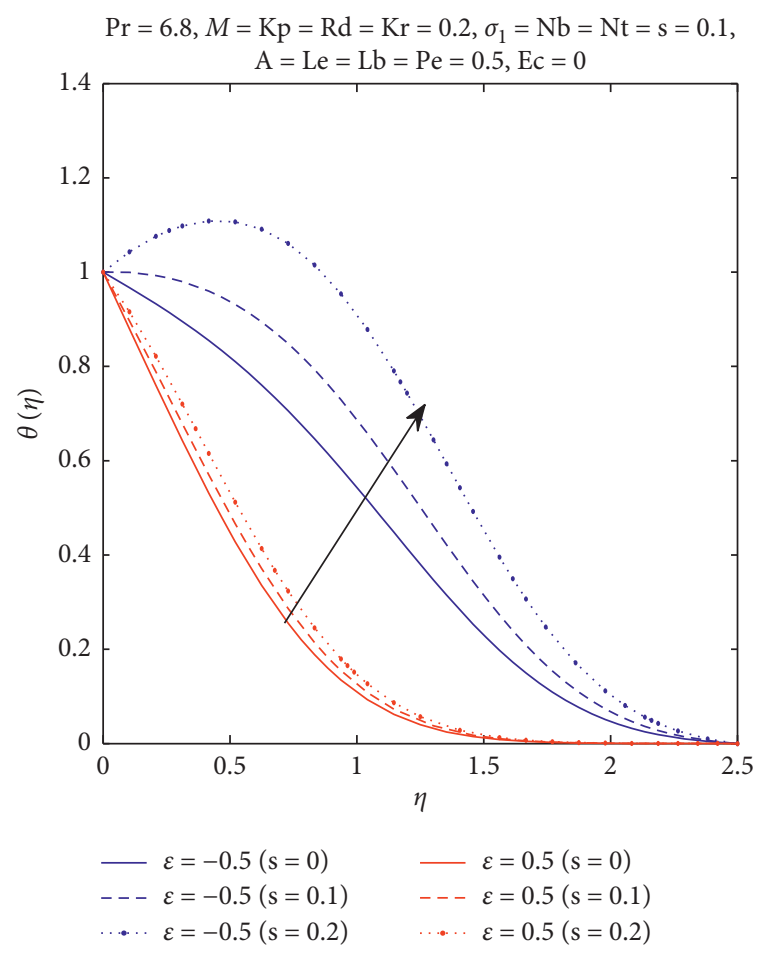

FIgURE 12: Temperature profile $\theta(\eta)$ for different $s$.

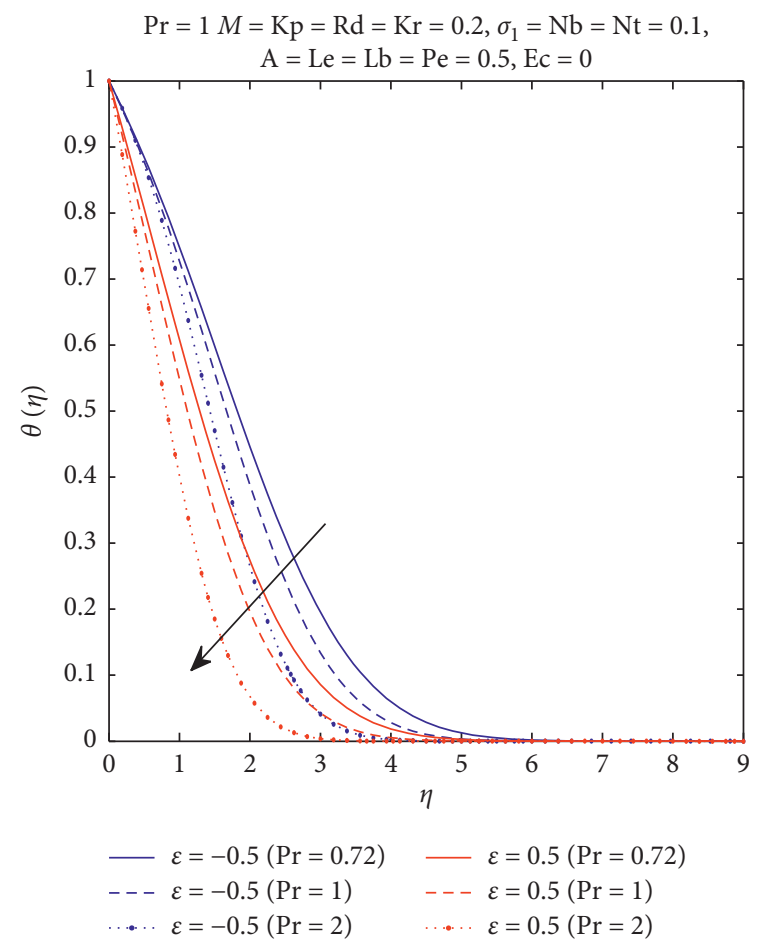

Figure 13: Temperature profile $\theta(\eta)$ for different Pr.

Figure 15 represents the influence of the Peclet number Pe on the density of motile microorganisms profile. It is validated the fact that increment in Peclet number Pe causes 


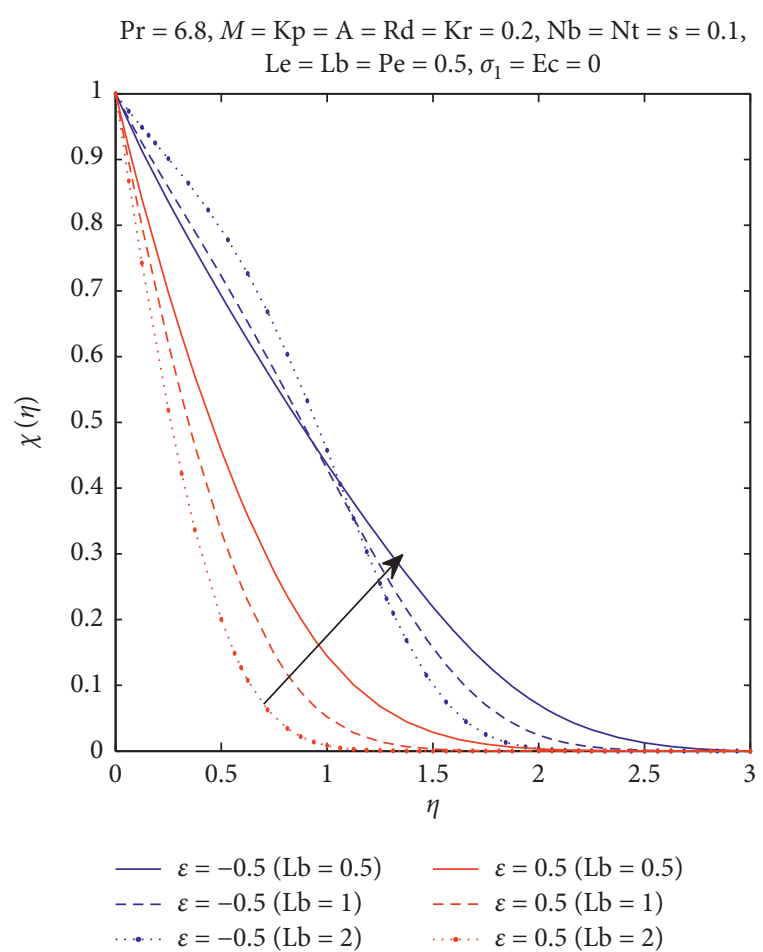

FIgURE 14: Microorganisms profile $\chi(\eta)$ for different Lb.

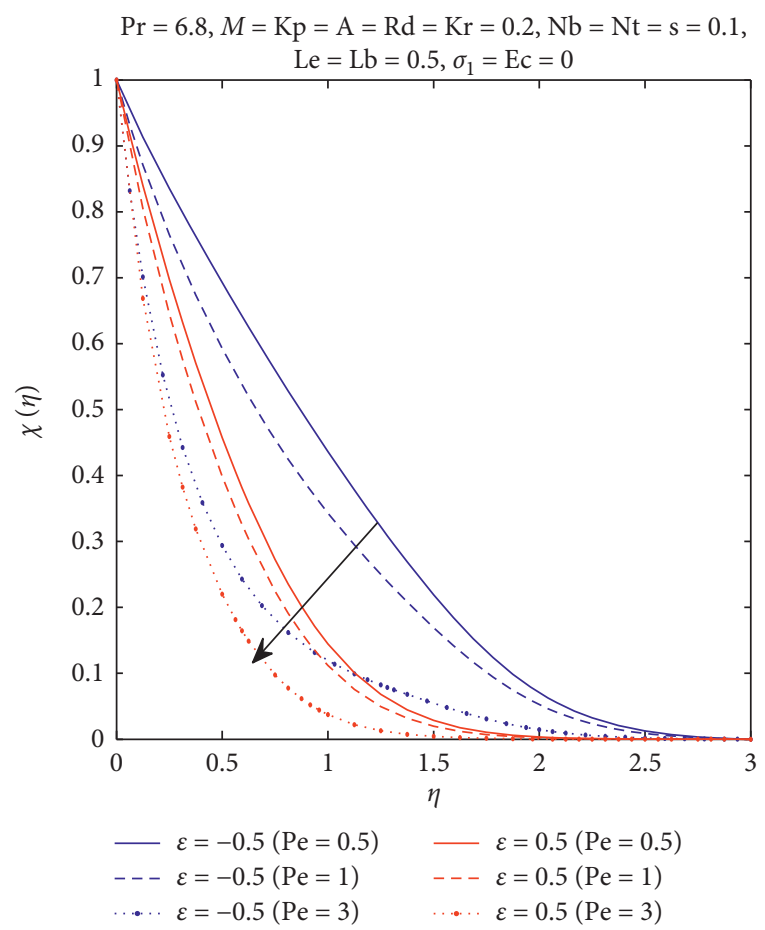

Figure 15: Microorganisms profile $\chi(\eta)$ for different Pe.

a reduction in motile microorganisms boundary layer thickness.

Figures 16 and 17 portray the impact of the Lewis number Le and the chemical reaction $\mathrm{Kr}$ on the concentration profile. It is analyzed that by increasing both the

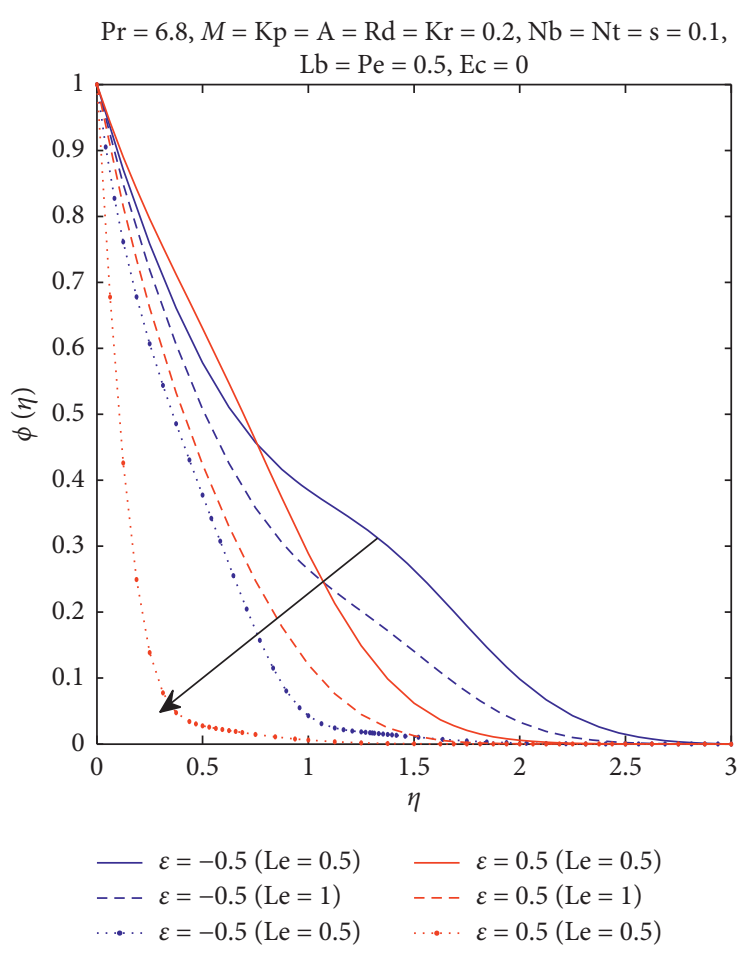

Figure 16: Concentration profile $\phi(\eta)$ for different Le.

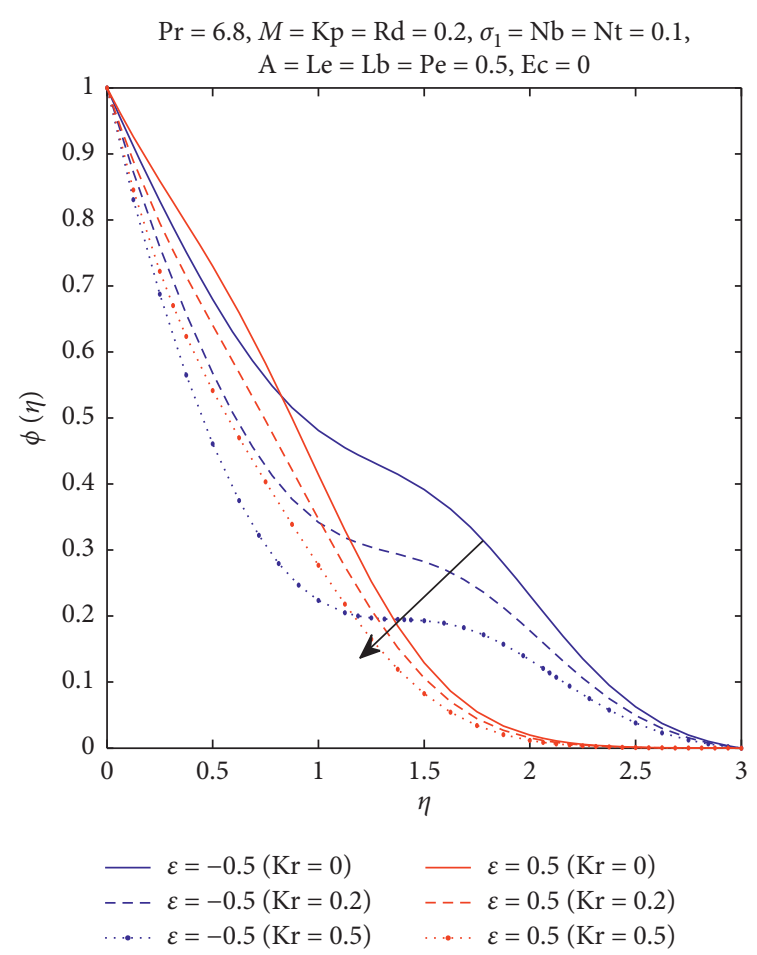

FIgURE 17: Concentration profile $\phi(\eta)$ for different $\mathrm{Kr}$.

parameter Lewis number Le and chemical reaction $\mathrm{Kr}$, the concentration boundary layer thins.

Figure 18 depicts the skin friction coefficient against the porosity parameter $K_{p}$ with variations $A$ and $M$. The skin 


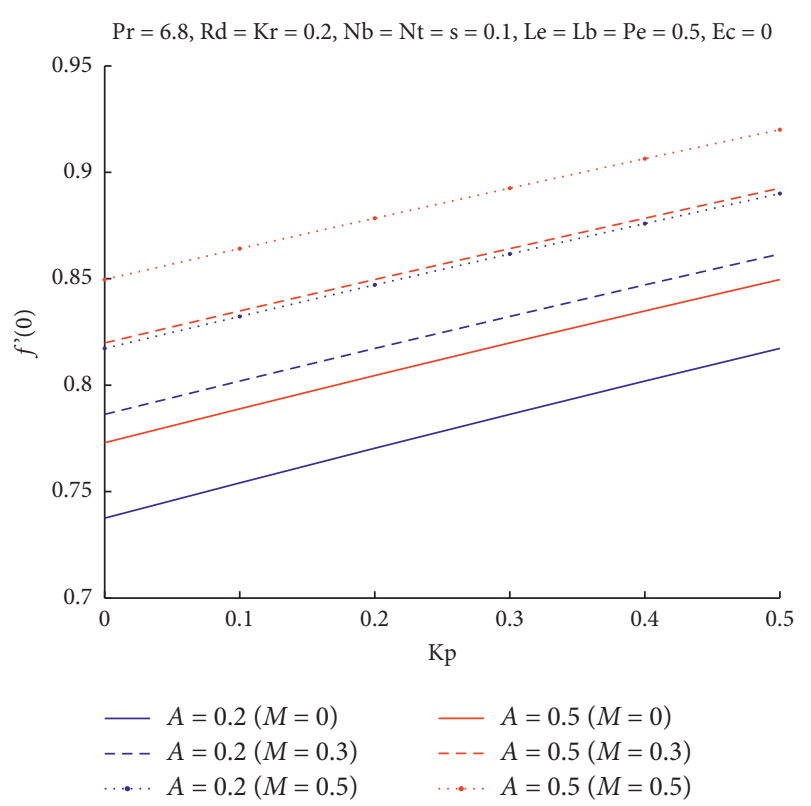

FIGURE 18: The skin friction coefficient with variations of $\mathrm{A}$ and $\mathrm{M}$.

friction seems to increase with the porosity parameter and with the increasing values of $A$ and $M$.

\section{Conclusions}

The current analysis focuses on the unsteady MHD stagnation point flow of bionanofluid with internal heat generation/absorption in a permeable medium with thermal radiation and chemical reaction into account over a stretching and shrinking sheet. The significant findings of the problem are summarized as follows:

(1) The skin friction coefficient enhances for higher values of the unsteady parameter $A$, magnetic parameter $M$, and porosity parameter Kp.

(2) The increment in the Brownian motion parameter $\mathrm{Nb}$, thermophoresis parameter $\mathrm{Nt}$, thermal radiation parameter Rd, Eckert number Ec, heat source parameter $s$ causes enhancement in thermal boundary layer thickness while an increase in Prandtl number Pr causes a reduction in thermal boundary layer thickness.

(3) The concentration boundary layer thickness increases for the thermophoresis parameter $\mathrm{Nt}$, whereas it decreases for higher values of the Brownian motion parameter $\mathrm{Nb}$, Lewis number Le, and chemical reaction parameter $\mathrm{Kr}$.

(4) The increment of the Brownian motion parameter $\mathrm{Nb}$, bioconvection parameter $\mathrm{Lb}$, and Peclet number $\mathrm{Pe}$ reduces the density of motile microorganisms while it increases for larger values of the thermophoresis parameter $\mathrm{Nt}$.

(5) Different trends have been seen for boundary layer thickness through graphs. Graphs describe that boundary layer thickness is different in the stretching sheet case when compared to the shrinking sheet case.
(6) The skin friction coefficient increases with the increase in porosity parameter $K_{P}$ as it can be seen through tables and graphical representation.

\section{Nomenclature}

a: $\quad$ Positive constant $\left(\mathrm{s}^{-1}\right)$

$(u, v)$ : The velocity components $\left(\mathrm{ms}^{-1}\right)$

$(x, \quad$ Cartesian coordinates $(\mathrm{m})$

$y):$

A: Unsteadiness parameter

$A_{1}$ : Dimensionless parameter

$\beta_{o}: \quad$ Applied magnetic field $\left(\mathrm{Nm}^{-1} \mathrm{~A}^{-1}\right)$

$\mu$ : The coefficient of viscosity (Pas)

$\rho: \quad$ The density of fluid $\left(\mathrm{kgm}^{-3}\right)$

$\sigma: \quad$ The electrical conductivity of the fluid $\left(\mathrm{S} \mathrm{m}^{-1}\right)$ (S is siemens)

M: Magnetic parameter

Kp: Porosity parameter

$\epsilon$ : $\quad$ Stretching/Shrinking parameter

$T: \quad$ Fluid temperature $(\mathrm{K})$

$T_{w}$ : Constant temperature at wall $(\mathrm{K})$

$T_{\infty}$ : The ambient fluid temperature $(\mathrm{K})$

$k: \quad$ The thermal conductivity $\left(\mathrm{Wm}^{-1} \mathrm{~K}^{-1}\right)$

$\alpha: \quad$ The thermal diffusivity $\left(\mathrm{m}^{2} \mathrm{~s}^{-1}\right)$

$k_{1}$ : $\quad$ Mean absorption coefficient $\left(\mathrm{m}^{-1}\right)$

$\sigma^{*}: \quad$ Stefan-Boltzman constant $\left(\mathrm{Wm}^{-2} \mathrm{~K}^{-4}\right)$

$C_{p}: \quad$ The specific heat capacity $\left(\mathrm{Jkg}^{-1} \mathrm{~K}^{-1}\right)$

$q_{r}$ : The radiative heat flux $\left(\mathrm{Wm}^{-2}\right)$

Q: $\quad$ Rate of heat generation/absorption

$C_{f}$ : Skin friction coefficient

$\mathrm{Nu}_{x}$ : Local Nusselt parameter

$s$ : Local heat source/sink parameter

Rd: Thermal radiation parameter

Pr: Ambient Prandtl number

$D_{B}: \quad$ Brownian diffusion coefficient $\left(\mathrm{m}^{2} \mathrm{~s}^{-1}\right)$

$D_{T}$ : Thermophoretic diffusion coefficient $\left(\mathrm{m}^{2} \mathrm{~s}^{-1}\right)$

$D_{m}: \quad$ Diffusivity of microorganisms $\left(\mathrm{m}^{2} \mathrm{~s}^{-1}\right)$

$D_{n}: \quad$ Diffusivity coefficient $\left(\mathrm{m}^{2} \mathrm{~s}^{-1}\right)$

$\tau_{1}$ : $\quad$ Ratio of effective heat capacitance of the nanoparticle to the base fluid

$(\rho c)_{p}$ : Nanoparticle heat capacity $\left(\mathrm{JK}^{-1} \mathrm{~m}^{3}\right)$

$N_{b}: \quad$ Brownian motion parameter

$N_{t}$ : Thermophoresis parameter

$C: \quad$ The concentration

$C_{w}: \quad$ The concentration at the wall

$C_{\infty}$ : The ambient fluid concentration

$N$ : The concentration of microorganisms

$N_{w}$ : Microorganisms at the wall

$N_{\infty}$ : Microorganisms far from the wall

Le: Lewis number

Lb: Bioconvection Lewis number

$b$ : Chemotaxis constant (m)

$w_{c}$ : $\quad$ Maximum cell swimming speed $\mathrm{ms}^{-1}$

Pe: Peclet number

$\mathrm{Sh}_{x}$ : Local Sherwood parameter

$\mathrm{Nn}_{x}$ : Local density parameter of the motile microorganisms. 


\section{Data Availability}

No experimental data were used to support this study.

\section{Conflicts of Interest}

The authors declare that they have no conflicts of interest.

\section{Acknowledgments}

ZHS would like to thank the Department of Mathematics, University of the Punjab, Lahore, for the partial support. AM would like to acknowledge the support of Mathematics Teaching and Learning, Research Groups within the Department of Mathematics, FLU, Nord University, Bodø.

\section{References}

[1] Q. Z. Xue, "Model for effective thermal conductivity of nanofluids," Physics Letters A, vol. 307, no. 5-6, pp. 313-317, 2003.

[2] W. Yu and S. U. S. Choi, "The role of interfacial layers in the enhanced thermal conductivity of nanofluids: a renovated Maxwell model," Journal of Nanoparticle Research, vol. 5, no. 1-2, pp. 167-171, 2003.

[3] W. Yu and S. U. S. Choi, "The role of interfacial layers in the enhanced thermal conductivity of nanofluids: a renovated Hamilton?Crosser model," Journal of Nanoparticle Research, vol. 6, no. 4, pp. 355-361, 2004.

[4] https://tfaws.nasa.gov/TFAWS10/Proceedings/Interdisciplinary/ Debjyoti\%20Banerjee.pdf.

[5] X.-Q. Wang and A. S. Mujumdar, "Heat transfer characteristics of nanofluids: a review," International Journal of Thermal Sciences, vol. 46, no. 1, pp. 1-19, 2007.

[6] T. J. Pedley, N. A. Hill, and J. O. Kessler, "The growth of bioconvection patterns in a uniform suspension of gyrotactic micro-organisms," Journal of Fluid Mechanics, vol. 195, no. 1, pp. 223-237, 1988.

[7] W. A. Khan and O. D. Makinde, "MHD nanofluid bioconvection due to gyrotactic microorganisms over a convectively heat stretching sheet," International Journal of Thermal Sciences, vol. 81, pp. 118-124, 2014.

[8] A. V. Kuznetsov, A. A. Avramenko, and P. Geng, "Analytical investigation of a falling plume caused by bioconvection of oxytactic bacteria in a fluid saturated porous medium," International Journal of Engineering Science, vol. 42, no. 5-6, pp. 557-569, 2004.

[9] W. N. Mutuku and O. D. Makinde, "Hydromagnetic bioconvection of nanofluid over a permeable vertical plate due to gyrotactic microorganisms," Computers \& Fluids, vol. 95, pp. 88-97, 2014.

[10] K. Naganthran, M. F. M. Basir, S. O. Alharbi, R. Nazar, A. M. Alwatban, and I. Tlili, "Stagnation point flow with timedependent bionanofluid past a sheet: richardson extrapolation technique," Processes, vol. 7, no. 10, p. 722, 2019.

[11] K. Zaimi, A. Ishak, and I. Pop, "Stagnation-point flow toward a stretching/shrinking sheet in a nanofluid containing both nanoparticles and gyrotactic microorganisms," Journal of Heat Transfer, vol. 136, no. 4, 2014.

[12] F. Ali and A. Zaib, "Unsteady flow of an eyring-powell nanofluid near stagnation point past a convectively heated stretching sheet," Arab Journal of Basic and Applied Sciences, vol. 26, no. 1, pp. 215-224, 2019.
[13] L. Zeng and T. J. Pedley, "Distribution of gyrotactic microorganisms in complex three-dimensional flows. Part 1. Horizontal shear flow past a vertical circular cylinder," Journal of Fluid Mechanics, vol. 852, pp. 358-397, 2018.

[14] N. A. Shah, A. H. Seikh, I. Tlili et al., "Natural convection of bio-nanofluid between two vertical parallel plates with damped shear and thermal flux," Journal of Molecular Liquids, vol. 296, p. 111575, 2019.

[15] N. A. Amirsom, M. J. Uddin, M. F. Md Basir, A. Kadir, O. A. Bég, and A. I. Md. Ismail, "Computation of melting dissipative magnetohydrodynamic nanofluid bioconvection with second-order slip and variable thermophysical properties," Applied Sciences, vol. 9, no. 12, p. 2493, 2019.

[16] A. M. Khdher, N. A. C. Sidik, W. A. W. Hamzah, and R. Mamat, "An experimental determination of thermal conductivity and electrical conductivity of bio glycol based $\mathrm{Al}$ 2 O 3 nanofluids and development of new correlation," International Communications in Heat and Mass Transfer, vol. 73, pp. 75-83, 2016.

[17] M. M. Bhatti and E. E. Michaelides, "Study of Arrhenius activation energy on the thermo-bioconvection nanofluid flow over a Riga plate," Journal of Thermal Analysis and Calorimetry, pp. 1-10, 2020.

[18] S. M. Atif, S. Hussain, and M. Sagheer, "Magnetohydrodynamic stratified bioconvective flow of micropolar nanofluid due to gyrotactic microorganisms," AIP Advances, vol. 9, no. 2, Article ID 025208, 2019.

[19] T. Zhang, S. U. Khan, M. Imran, I. Tlili, H. Waqas, and N. Ali, "Activation energy and thermal radiation aspects in bioconvection flow of rate-type nanoparticles configured by a stretching/shrinking disk," Journal of Energy Resources Technology, vol. 142, no. 11, 2020.

[20] Y. Li, H. Waqas, M. Imran, U. Farooq, F. Mallawi, and I. Tlili, "A numerical exploration of modified second-grade nanofluid with motile microorganisms, thermal radiation, and Wu's slip," Symmetry, vol. 12, no. 3, p. 393, 2020.

[21] A. M. Alwatban, S. U. Khan, H. Waqas, and I. Tlili, "Interaction of Wu's slip features in bioconvection of eyring powell nanoparticles with activation energy," Processes, vol. 7, no. 11, p. 859, 2019.

[22] Y. Wang, H. Waqas, M. Tahir, M. Imran, and C. Y. Jung, "Effective Prandtl aspects on bio-convective thermally developed magnetized tangent hyperbolic nanoliquid with gyrotactic microorganisms and second order velocity slip," IEEE Access, vol. 7, pp. 130008-130023, 2019.

[23] M. Izadi, M. A. Sheremet, S. A. M. Mehryan, I. Pop, H. F. Öztop, and N. Abu-Hamdeh, "MHD thermogravitational convection and thermal radiation of a micropolar nanoliquid in a porous chamber," International Communications in Heat and Mass Transfer, vol. 110, p. 104409, 2020.

[24] Y. S. Daniel, Z. A. Aziz, Z. Ismail, and F. Salah, "Entropy analysis in electrical magnetohydrodynamic (MHD) flow of nanofluid with effects of thermal radiation, viscous dissipation, and chemical reaction," Theoretical and Applied Mechanics Letters, vol. 7, no. 4, pp. 235-242, 2017.

[25] T. Muhammad, H. Waqas, S. A. Khan, R. Ellahi, and S. M. Sait, "Significance of nonlinear thermal radiation in 3D Eyring-Powell nanofluid flow with Arrhenius activation energy," Journal of Thermal Analysis and Calorimetry, pp. 1-16, 2020.

[26] M. Sohail, R. Naz, and S. I. Abdelsalam, "On the onset of entropy generation for a nanofluid with thermal radiation and gyrotactic microorganisms through 3D flows," Physica Scripta, vol. 95, no. 4, Article ID 045206, 2020. 
[27] B. J. Gireesha, G. Sowmya, M. I. Khan, and H. F. Öztop, “Flow of hybrid nanofluid across a permeable longitudinal moving fin along with thermal radiation and natural convection," Computer Methods and Programs in Biomedicine, vol. 185, p. 105166, 2020.

[28] M. R. Eid, "Chemical reaction effect on MHD boundary-layer flow of two-phase nanofluid model over an exponentially stretching sheet with a heat generation," Journal of Molecular Liquids, vol. 220, pp. 718-725, 2016.

[29] R. S. Tripathy, G. C. Dash, S. R. Mishra, and S. Baag, "Chemical reaction effect on MHD free convective surface over a moving vertical plate through porous medium," Alexandria Engineering Journal, vol. 54, no. 3, pp. 673-679, 2015.

[30] D. Pal and B. Talukdar, "Buoyancy and chemical reaction effects on MHD mixed convection heat and mass transfer in a porous medium with thermal radiation and Ohmic heating," Communications in Nonlinear Science and Numerical Simulation, vol. 15, no. 10, pp. 2878-2893, 2010.

[31] H. R. Kataria and H. R. Patel, "Radiation and chemical reaction effects on MHD Casson fluid flow past an oscillating vertical plate embedded in porous medium," Alexandria Engineering Journal, vol. 55, no. 1, pp. 583-595, 2016.

[32] Z. Shah, P. Kumam, and W. Deebani, "Radiative MHD Casson Nanofluid Flow with Activation energy and chemical reaction over past nonlinearly stretching surface through entropy generation," Scientific Reports, vol. 10, no. 1, pp. 1-14, 2020.

[33] G. Rasool, T. Zhang, A. J. Chamkha, A. Shafiq, I. Tlili, and G. Shahzadi, "Entropy generation and consequences of binary chemical reaction on MHD Darcy-forchheimer Williamson nanofluid flow over non-linearly stretching surface," Entropy, vol. 22, no. 1, p. 18, 2020.

[34] U. Khan, A. Zaib, I. Khan, and K. S. Nisar, "Activation energy on MHD flow of titanium alloy (Ti6Al4V) nanoparticle along with a cross flow and streamwise direction with binary chemical reaction and non-linear radiation: dual Solutions," Journal of Materials Research and Technology, vol. 9, no. 1, pp. 188-199, 2020.

[35] M. I. Khan, S. A. Khan, T. Hayat, S. Qayyum, and A. Alsaedi, "Entropy generation analysis in MHD flow of viscous fluid by a curved stretching surface with cubic autocatalysis chemical reaction," The European Physical Journal Plus, vol. 135, no. 2, pp. 1-17, 2020.

[36] M. Khan, T. Salahuddin, M. Y. Malik, A. Tanveer, A. Hussain, and A. S. Alqahtani, "3-D axisymmetric Carreau nanofluid flow near the Homann stagnation region along with chemical reaction: application Fourier's and Fick's laws," Mathematics and Computers in Simulation, vol. 170, pp. 221-235, 2020.

[37] P. P. Gharami, S. Reza-E-Rabbi, S. M. Arifuzzaman, M. S. Khan, T. Sarkar, and S. F. Ahmmed, "MHD effect on unsteady flow of tangent hyperbolic nano-fluid past a moving cylinder with chemical reaction," SN Applied Sciences, vol. 2, no. 7, pp. 1-16, 2020.

[38] A. Hamid, M. Hashim, and M. Khan, "Impacts of binary chemical reaction with activation energy on unsteady flow of magneto-Williamson nanofluid," Journal of Molecular Liquids, vol. 262, pp. 435-442, 2018.

[39] P. S. Reddy, P. Sreedevi, and A. J. Chamkha, "MHD boundary layer flow, heat and mass transfer analysis over a rotating disk through porous medium saturated by $\mathrm{Cu}$-water and $\mathrm{Ag}$-water nanofluid with chemical reaction," Powder Technology, vol. 307, pp. 46-55, 2017.
[40] B. Ali, Y. Nie, S. A. Khan, M. T. Sadiq, and M. Tariq, "Finite element simulation of multiple slip effects on MHD unsteady maxwell nanofluid flow over a permeable stretching sheet with radiation and thermo-diffusion in the presence of chemical reaction," Processes, vol. 7, no. 9, p. 628, 2019.

[41] M. R. Krishnamurthy, B. C. Prasannakumara, B. J. Gireesha, and R. S. R. Gorla, "Effect of chemical reaction on MHD boundary layer flow and melting heat transfer of Williamson nanofluid in porous medium," Engineering Science and Technology, an International Journal, vol. 19, no. 1, pp. 53-61, 2016.

[42] M. Mustafa, J. A. Khan, T. Hayat, and A. Alsaedi, "Buoyancy effects on the MHD nanofluid flow past a vertical surface with chemical reaction and activation energy," International Journal of Heat and Mass Transfer, vol. 108, pp. 1340-1346, 2017.

[43] P. B. A. Reddy, "Magnetohydrodynamic flow of a Casson fluid over an exponentially inclined permeable stretching surface with thermal radiation and chemical reaction," Ain Shams Engineering Journal, vol. 7, no. 2, pp. 593-602, 2016.

[44] I. Ullah, K. Bhattacharyya, S. Shafie, and I. Khan, "Unsteady MHD mixed convection slip flow of Casson fluid over nonlinearly stretching sheet embedded in a porous medium with chemical reaction, thermal radiation, heat generation/ absorption and convective boundary conditions," PloS One, vol. 11, no. 10, Article ID e0165348, 2016.

[45] G. T. Thammanna, K. Ganesh Kumar, B. J. Gireesha, G. K. Ramesh, and B. C. Prasannakumara, "Three dimensional MHD flow of couple stress Casson fluid past an unsteady stretching surface with chemical reaction," Results in Physics, vol. 7, pp. 4104-4110, 2017.

[46] M. M. Bhatti, S. R. Mishra, T. Abbas, and M. M. Rashidi, “A mathematical model of MHD nanofluid flow having gyrotactic microorganisms with thermal radiation and chemical reaction effects," Neural Computing and Applications, vol. 30, no. 4, pp. 1237-1249, 2018.

[47] M. A. Imran, F. Miraj, I. Khan, and I. Tlili, "MHD fractional Jeffrey's fluid flow in the presence of thermo diffusion, thermal radiation effects with first order chemical reaction and uniform heat flux," Results in Physics, vol. 10, pp. 10-17, 2018.

[48] S. A. Khan, Y. Nie, and B. Ali, "Multiple slip effects on magnetohydrodynamic axisymmetric buoyant nanofluid flow above a stretching sheet with radiation and chemical reaction," Symmetry, vol. 11, no. 9, p. 1171, 2019.

[49] N. V. Ganesh, Q. M. Al-Mdallal, S. Al Fahel, and S. Dadoa, "Riga-Plate flow of $\gamma \mathrm{Al} 2 \mathrm{O} 3$-water/ethylene glycol with effective Prandtl number impacts," Heliyon, vol. 5, no. 5, Article ID e01651, 2019.

[50] N. V. Ganesh, Q. M. Al-Mdallal, K. Reena, and S. Aman, "Blasius and Sakiadis slip flow of H2O-C2H6O2 (50:50) based nanoliquid with different geometry of boehmite alumina nanoparticles," Case Studies in Thermal Engineering, vol. 16, p. 100546, 2019.

[51] W. Ibrahim, B. Shankar, and M. M. Nandeppanavar, "MHD stagnation point flow and heat transfer due to nanofluid towards a stretching sheet," International Journal of Heat and Mass Transfer, vol. 56, no. 1-2, pp. 1-9, 2013.

[52] J. Kierzenka and L. F. Shampine, "A BVP solver based on residual control and the Maltab PSE," ACM Transactions on Mathematical Software, vol. 27, no. 3, pp. 299-316, 2001.

[53] J. Kierzenka and L. F. Shampine, "A BVP solver that controls residual and error," Journal of the Society for Industrial and Applied Mathematics, vol. 3, no. 1-2, pp. 27-41, 2008. 